\title{
A GENERALIZED REVIEW ON HOMOGENIZATION AND TWO-SCALE CONVERGENCE
}

\section{GÉRARD ZONGO, OUSSÉNI SO, GENEVIÈVE BARRO and BLAISE SOMÉ}

Laboratoire d'Analyse Numérique, d'Informatique

et de Biomathématique

Unité de Formation et de Recherche-Sciences

Exactes et Appliquées

Université Joseph KI-ZERBO

12 BP 55 Ouaga 12

Burkina Faso

e-mail: gerardzongo1@gmail.com

soousseni@gmail.com

barro_genevieve@yahoo.fr

blaisesomeouaga1@gmail.com

\begin{abstract}
We study homogenization and two-scale convergence. Homogenization is a mathematical concept that makes it possible to develop a global model of the behaviour of a physical structure evolving in a heterogeneous structure. The behaviour of this physical structure will therefore be studied in a homogeneous environment, which greatly facilitates calculations. The two-scale convergence method was introduced by Nguetseng and later developed by Allaire. It is a particular form of weak convergence, a convergence between weak convergence
\end{abstract}

2010 Mathematics Subject Classification: 35B27, 76M50, 34E10.

Keywords and phrases: two-scale convergence, multiscale convergence, weak convergence, strong convergence, homogenization.

Received July 16, 2019; Revised August 27, 2019

(C) 2019 Scientific Advances Publishers 
and strong convergence. The two-scale convergence simplifies the proof of homogenization theory. The method evolved very quickly and has been extended to several cases depending on the functional space.

\section{Introduction}

The objective of this article is to present a general review on the theme of homogenization and two-scale convergence.

The idea of homogenization was introduced by Ivo M. Babuska in 1974, see [14-16]. After, it has been studied by many other authors. We can cite Bensoussan et al. in 1978 [19], Srinivasan Kesavan in 1979 [47], Sanchez-Palencia in 1980 [63], Bakhvalov and Panasenko in 1989 [18].

Homogenization is a mathematical theory that consists in replacing a very heterogeneous medium by a homogeneous medium which is easy to study. The two-scale convergence gives a rigourous mathematical justification of homogenization. It has been defined following an idea of Nguetseng [55] in the $L^{2}$ space. In 1992, Allaire renamed it in «Twoscale convergence». Allaire set out the essential properties and applied it to several homogenization problems. This convergence has been extended to the multiscale case by Ene and Saint Jean Paulin in 1995 [37], and by Allaire and Briane in 1996 [6]. The periodic unfolding method was introduced in 2002 by Cioranescu et al. [29, 30, 36]. It generalises the notion of multiscale convergence. Nguetseng extended the theory to the Sobolev space $W^{1,2}$ in 2002 [49]. In 2003, he extended it to the non periodic case ( $\sum$-convergence) [56-58].

Before introducing these two notions, we will dwell on the functional spaces and the notations which intervene here.

The classic notation for a periodic function space consists in using $\sharp$ hashtag.

$(0, T)$ is an interval with $T>0$.

$\Omega$ : periodic domain of $\mathbb{R}^{N}$. 
Let's be $\mathbb{R}_{y}^{N}$ (N integer $\left.\geqslant 1\right)$ the numerical space on $\mathbb{R}^{N}$ of variables (called microscopic) $y=\left(y_{1}, \ldots, y_{N}\right), \mathbb{R}_{x}^{N}$ the numerical space on $\mathbb{R}^{N}$ of variables (called macroscopic) $x=\left(x_{1}, \ldots, x_{N}\right)$ and $Y=\left(-\frac{1}{2} ; \frac{1}{2}\right)$ the open unit cube in $\mathbb{R}_{y}^{N}$.

$\mathbb{R}_{y}^{N}$ : with Lebesgue's measure.

$Y$ : unit cell.

$\mathcal{C}(\bar{\Omega})$ is the space of continuous functions on $\bar{\Omega}$.

$L^{p}(\Omega)=\left\{f: \Omega \rightarrow \mathbb{R}\right.$ measurable $\left.:\|f\|_{p}=\left(\int_{\Omega}\|f\|^{p} d x\right)^{\frac{1}{p}}<\infty\right\}$.

$C_{\sharp}^{0}(Y)$ is a set of continuous functions and $Y$-periodic on $\mathbb{R}^{N}$.

$C_{\sharp}^{k}(Y)$ is a set of functions $C^{k}$-continuous and $Y$-periodic on $\mathbb{R}^{N}$.

$L_{\sharp}^{p}(Y)$ for $1 \leqslant p<\infty$ fixed, is the space of functions $w \in L_{\mathrm{loc}}^{p}\left(\mathbb{R}_{y}^{N}\right)$

which is $Y$-periodic, i.e., which verifies $w(y+k)=w(y)$ a.a. $y \in \mathbb{R}^{N}$, for all $k \in \mathbb{Z}^{N}$.

$L_{\sharp}^{2}(Y) / \mathbb{R}$ is the set of functions $u(y)$, in $L^{2}(Y), \quad Y$-periodic and defined to the nearest constant.

$W_{\sharp}^{1, p}(Y)$ denotes the subset of $W^{1, p}(Y)$ of all functions $u$ with mean value zero which have the same trace on opposite faces of $Y$.

$H_{\sharp}^{1}(Y)$ is the space of function $H_{\text {loc }}^{1}\left(\mathbb{R}^{N}\right)$ and $Y$-periodic.

$H^{-k}\left(\mathbb{R}^{n}\right)$ is the space of linear forms $u$ on $H^{k}\left(\mathbb{R}^{n}\right)$. 
$L^{2}((0, T) \times \Omega)$ is the set of functions $u(t, x)$ of square summable in $(0, T) \times \Omega$.

$L^{1}\left(\Omega ; C_{\sharp}(Y)\right)$ is the space of functions $f: \Omega \rightarrow C_{\sharp}(Y)$ which are measurable and satisfy that $\int_{\Omega}\|f(x)\|_{C_{\sharp}(Y)} d x<\infty$.

$L^{2}\left(\Omega ; H_{\sharp}^{1}(Y) / \mathbb{R}\right)$ is the set of summable square functions in $\Omega$ with values in $H_{\sharp}^{1}(Y) / \mathbb{R}$.

$\mathcal{C}^{\infty}(\Omega)$ : the space of functions indefinitely differentiable on $\Omega$ where each derivative is continuous on $\Omega$.

$\mathcal{D}\left(\Omega ; C_{\sharp}^{\infty}(Y)\right)$ is the space of functions indefinitely regular with compact support in $\Omega$ to values in the space $C_{\sharp}^{\infty}(Y)$.

We say that a function $f: \Omega \rightarrow \mathbb{C}$ is with compact support if there is a compact $K \subseteq \Omega$ such that $f=0$ on $\Omega \backslash K$.

$\mathcal{C}_{c}(\Omega)$ is the space of continuous functions on $\Omega$ with a compact support.

$\mathcal{C}_{c}^{\infty}(\Omega)=\mathcal{C}^{\infty}(\Omega) \cap \mathcal{C}_{c}(\Omega) \quad$ (some authors write $\mathcal{D}(\Omega)$ instead of $\left.\mathcal{C}_{c}^{\infty}(\Omega)\right)$ : the space of functions indefinitely differentiable on $\Omega$ with compact support in $\Omega$.

After this introduction, in the Section 2, we will define the theory of homogenization and give simple example of homogenized problem. In Section 3, we will define two-scale convergence, give some examples of problems solved with this theory and an example of solving problem with this theory.

We will end this survey by a conclusion in Section 4 . 


\section{Homogenization Theory}

The classical method of asymptotic analysis by homogenization is the asymptotic development method described, for example, in [17, 19, 62]. It is a formal method which does not provide a convergence result to the homogenized model but which, nevertheless, makes it possible to have an explicit form of this model and its effective parameters.

\subsection{Problem position}

We consider a diffusion or conductivity model in a periodic medium (see Figure 1). Let $\Omega$ periodic domain of $\mathbb{R}^{N}$ with $N \geqslant 1$. Let's note by $\varepsilon$ the period of the domain (a positive number which is supposed to be very small compared to the size of the domain), the periodic cell of the rescaled unit $Y=(0,1)^{N}$. Conductivity isn't constant in $\Omega$, but varies with a period $\varepsilon$ in each direction. We note by $A(y)$ the second order

tensor matrix, where $y=\frac{x}{\varepsilon} \in Y$ is the rapid variable also called microscopic variable, while $x \in \Omega$ is the slow variable also called macroscopic variable.

Let $A$ be a tensor under the classic tensor assumptions, namely the existence of $0<c<C$ such that

$$
\forall \xi \in \mathbb{R}^{N}, c|\xi|^{2} \leqslant(A \xi, \xi) \leqslant C|\xi|^{2}
$$

We note by $f(x) \in L^{2}(\Omega)$ the source term. The conductivity equation is as follows:

$$
\begin{cases}-\operatorname{div}\left(A\left(\frac{x}{\varepsilon}\right) \nabla u_{\varepsilon}\right) & =f \text { in } \Omega, \\ u_{\varepsilon} & =0 \text { on } \partial \Omega .\end{cases}
$$

$u_{\varepsilon}$ is an unknown function. 
The domain is represented below

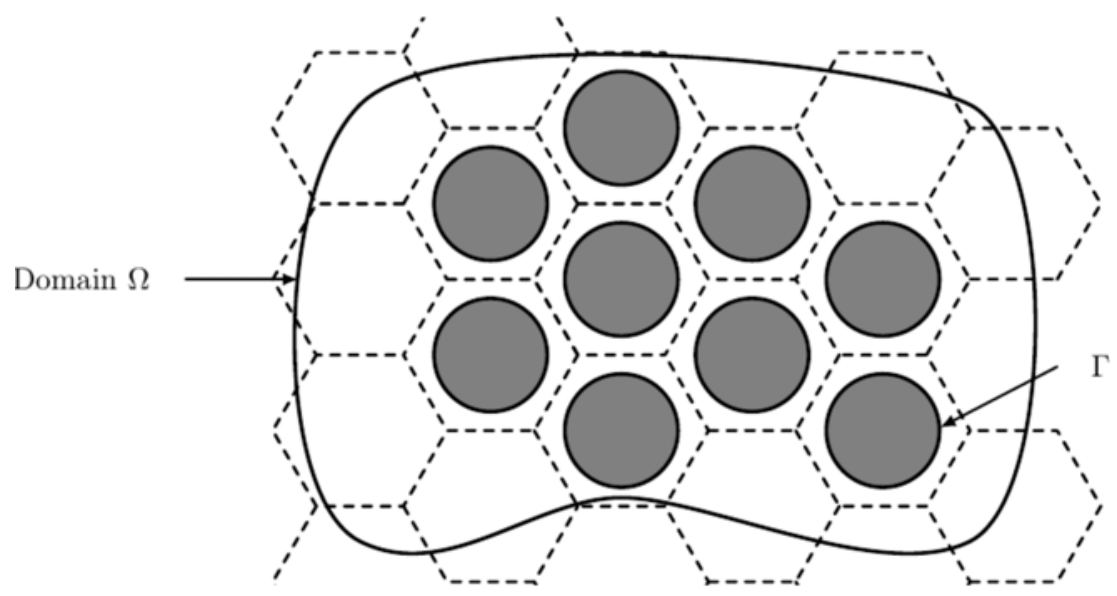

Figure 1. A periodic domain.

Remark 1. The problem (2) is well posed because $f \in L^{2}$, therefore according to Lax-Milgram's lemma, there is a unique solution $u_{\varepsilon} \in H_{0}^{1}(\Omega)$. In addition, the estimation energy gives

$$
\left\|u_{\varepsilon}\right\|_{L^{2}(\Omega)}+\left\|\nabla u_{\varepsilon}\right\|_{L^{2}(\Omega)} \leqslant C,
$$

where $C$ is a constant independent of $\varepsilon$.

There are different methodologies between the traditional physical approach to homogenization and the mathematical theory of homogenization. In literature, the so-called representative volume element (RVE) method is often used (see [28] or Chapter 1 in [45]).

In the sub-section below, we will give an example of mathematical homogenization in a periodic medium.

\subsection{Ansatz}

The asymptotic expansion method is a heuristic method $^{1}$; for further clarification, see [18, 19,63]. As previously stated, the starting point is asymptotic two-scale expansion, for a solution $u_{\varepsilon}$ of the Equation (2)

$1_{a}$ heuristic is a calculation method that provides a fast (in polynomial time) feasible, not necessarily optimal, solution for an optimization problem. 


$$
\begin{aligned}
& u_{\varepsilon}(x)=\sum_{i=0}^{+\infty} \varepsilon^{i} u_{i}\left(x, \frac{x}{\varepsilon}\right) \\
& u_{\varepsilon}(x)=u_{0}\left(x, \frac{x}{\varepsilon}\right)+\varepsilon u_{1}\left(x, \frac{x}{\varepsilon}\right)+\varepsilon^{2} u_{2}\left(x, \frac{x}{\varepsilon}\right)+\cdots,
\end{aligned}
$$

where $u_{i}(x, y)$ is a function of two variables $x$ and $y$, periodic in $y$ with a period $Y=(0,1)^{N}$ ( $u_{i}$ is called function $Y$-periodic with respect to $y$ ). The derivative of $u_{i}$ and $u_{\varepsilon}$ gives:

$$
\nabla\left(u_{i}\left(x, \frac{x}{\varepsilon}\right)\right)=\left(\varepsilon^{-1} \nabla_{y} u_{i}+\nabla_{x} u_{i}\right)\left(x, \frac{x}{\varepsilon}\right),
$$

and

$$
\nabla u_{\varepsilon}(x)=\varepsilon^{-1} \nabla_{y} u_{0}\left(x, \frac{x}{\varepsilon}\right)+\sum_{i=0}^{+\infty} \varepsilon^{i}\left(\nabla_{y} u_{i+1}+\nabla_{x} u_{i}\right)\left(x, \frac{x}{\varepsilon}\right) .
$$

Applying that to the Equation (2) we obtain

$$
\begin{aligned}
& -\operatorname{div}\left(A(y)\left(\varepsilon^{-1} \nabla_{y} u_{0}(x, y)+\sum_{i=0}^{+\infty} \varepsilon^{i}\left(\nabla_{y} u_{i+1}+\nabla_{x} u_{i}\right)(x, y)\right)\right]=f(x) \\
& -\frac{1}{\varepsilon} \operatorname{div}_{y}\left[A(y)\left[\varepsilon^{-1} \nabla_{y} u_{0}(x, y)+\sum_{i=0}^{+\infty} \varepsilon^{i}\left(\nabla_{y} u_{i+1}+\nabla_{x} u_{i}\right)(x, y)\right]\right] \\
& -\operatorname{div}_{x}\left[A(y)\left[\varepsilon^{-1} \nabla_{y} u_{0}(x, y)+\sum_{i=0}^{+\infty} \varepsilon^{i}\left(\nabla_{y} u_{i+1}+\nabla_{x} u_{i}\right)(x, y)\right]\right] \\
& =f(x) .
\end{aligned}
$$

That gives the following series in $\varepsilon$ which will give a cascade equation:

$$
\begin{aligned}
& -\varepsilon^{-2} \operatorname{div}_{y}\left[A(y)\left[\nabla_{y} u_{0}(x, y)\right]\right] \\
& -\varepsilon^{-1} \operatorname{div}_{y}\left[A(y)\left[\left(\nabla_{y} u_{1}+\nabla_{x} u_{0}\right)(x, y)\right]\right]-\varepsilon^{-1} \operatorname{div}_{x}\left[A(y)\left[\nabla_{y} u_{0}(x, y)\right]\right]
\end{aligned}
$$




$$
\begin{aligned}
& -\varepsilon^{0}\left[\operatorname{div}_{y}\left[A(y)\left[\left(\nabla_{y} u_{2}+\nabla_{x} u_{1}\right)(x, y)\right]\right]+\operatorname{div}_{x}\left[A(y)\left[\left(\nabla_{y} u_{1}+\nabla_{x} u_{0}\right)(x, y)\right]\right]\right] \\
& -\sum_{i=0}^{+\infty} \varepsilon^{i}\left[\operatorname{div}_{y}\left[A(y)\left[\left(\nabla_{y} u_{i+2}+\nabla_{x} u_{i+1}\right)(x, y)\right]\right]\right. \\
& \left.+\operatorname{div}_{x}\left[A(y)\left[\left(\nabla_{y} u_{i+1}+\nabla_{x} u_{i}\right)(x, y)\right]\right]\right]=f(x) .
\end{aligned}
$$

To solve the cascade equations, we need a technical lemma:

Lemma 1 (Alternative of Fredholm ([5], p. 7)). Let $f$ be a function in $L_{\sharp}^{2}(Y)$ Y-periodic and $A$ a matrix Y-periodic, coercive and bounded (in norm $L^{\infty}$ ) of $\mathbb{R}^{N \times N}$, then the problem

$$
\begin{cases}-\operatorname{div}_{y} A(y) \nabla_{y} v(y)=f(y) & \text { in } Y \\ y \rightarrow v(y) & \text { is } Y \text {-periodic }\end{cases}
$$

admits a single solution up to an additive constant in $H_{\sharp}^{1}(Y) / \mathbb{R}$ if and only if

$$
\int_{Y} f(y) d y=0
$$

\section{Cascade equations:}

- The first equation to solve is:

$$
\begin{cases}\operatorname{div}_{y} A(y) \nabla_{y} u_{0}(x, y)=0 & \text { in } Y, \\ y \mapsto u_{0}(x, y) & \text { is } Y \text {-periodic. }\end{cases}
$$

In this equation $y$ is a variable and $x$ acts as a parameter. According to the Lemma 1, there is a single solution to this equation up to a constant. $u_{0}$ is therefore a function that does not depend on $y$, i.e., there is a function $u(x)$ such that

$$
u_{0}(x, y) \equiv u(x)
$$


- The second equation to solve is:

$$
\begin{cases}\left.\operatorname{div}_{y} A(y)\left(\nabla_{x} u_{0}(x, y)+\nabla_{y} u_{1}(x, y)\right)\right)=0 & \text { in } Y, \\ y \mapsto u_{1}(x, y) & \text { is } Y \text {-periodic. }\end{cases}
$$

This equation can be written as

$$
-\operatorname{div}_{y} A(y) \nabla_{y} u_{1}(x, y)=\operatorname{div}_{y} A(y) \nabla_{x} u_{0}(x, y),
$$

which gives

$$
-\operatorname{div}_{y} A(y) \nabla_{y} u_{1}(x, y)=\operatorname{div}_{y} A(y) \nabla_{x} u(x) .
$$

It's an equation of unknown $u_{1}$ in the periodic cell $Y$ and $u_{1}(x, y)$ depends linearly on the first derivative $\nabla_{x} u(x)$. By Lemma 1 , this equation also admits a unique solution up to a constant.

- The third equation to solve is:

$$
\begin{cases}\quad-\operatorname{div}_{y} A(y) \nabla_{y} u_{2}(x, y)=\operatorname{div}_{y} A(y) \nabla_{x} u_{1}(x, y) & \\ \quad+\operatorname{div}_{x} A(y)\left(\nabla_{x} u_{0}(x, y)+\nabla_{y} u_{1}(x, y)\right)+f(x) & \text { in } Y, \\ y \mapsto u_{2}(x, y) & \text { is } Y \text {-periodic. }\end{cases}
$$

This gives

$$
\begin{aligned}
-\operatorname{div}_{y} A(y) \nabla_{y} u_{2}(x, y)= & \operatorname{div}_{y} A(y) \nabla_{x} u_{1}(x, y) \\
& +\operatorname{div}_{x} A(y)\left(\nabla_{x} u(x)+\nabla_{y} u_{1}(x, y)\right)+f(x) .
\end{aligned}
$$

According to the Lemma 1, this problem in $u_{2}$ admits a unique solution up to a constant.

By integrating the right part of the Equation (8) on $Y$, and using the boundary condition for $u_{2}$, we get

$$
\int_{Y} \operatorname{div}_{y} A(y) \nabla_{y} u_{2}(x, y) d y=\int_{\partial Y}\left[A(y) \nabla_{y} u_{2}(x, y)\right] \cdot n d y=0,
$$


and

$$
-\operatorname{div}_{x}\left(\int_{Y} A(y)\left(\nabla_{x} u(x)+\nabla_{y} u_{1}(x, y)\right) d y\right)=f(x) \text { in } \Omega
$$

Since $u_{1}$ depends linearly on $\nabla_{x} u$, it corresponds to the following homogenized problem:

$$
\begin{cases}-\operatorname{div}_{x}\left(A^{*} \nabla_{x} u_{0}(x)\right) & =f(x) \text { in } \Omega \\ u_{0}(x) & =0 \text { on } \partial \Omega\end{cases}
$$

where $A^{*}$ is the homogenized conductivity matrix. Its coefficients are given by:

$$
A_{i, j}^{*}=\int_{Y} A(y)\left(e_{i}+\nabla_{y} \omega_{i}\right) \cdot\left(e_{j}+\nabla_{y} \omega_{j}\right) d y=\int_{Y} A(y)\left(e_{i}+\nabla_{y} \omega_{i}\right) \cdot e_{j} d y,
$$

where $\left(e_{i}\right)_{i}$ is the canonical basis of $\mathbb{R}^{n}$ and $w_{i}$ is the unique solution of the cell problem below

$$
\begin{cases}-\operatorname{div}_{y} A(y)\left(e_{i}+\nabla_{y} \omega_{i}(y)\right)=0 & \text { in } Y \\ y \mapsto \omega_{i}(y) & \text { is } Y \text {-periodic. }\end{cases}
$$

\subsection{Example of homogenized problem in one dimensional case} ([24], pp. 4-18)

The study of the monodimentional case is interesting because it makes it possible to calculate the exact solution and to see its evolution when the number of periods increases.

\subsubsection{Presentation of the problem}

Let a periodic elastic bar, that is to say constituted by the regular juxtaposition of $N_{c}$ identical patterns made up of two homogeneous 
materials. The stiffnesses are $k_{1}$ and $k_{2}$ of respective lengths $\ell_{1}$ and $\ell_{2}$. The pattern length is $\ell=\ell_{1}+\ell_{2}$ and the bar length is $L=N_{c} \ell$.

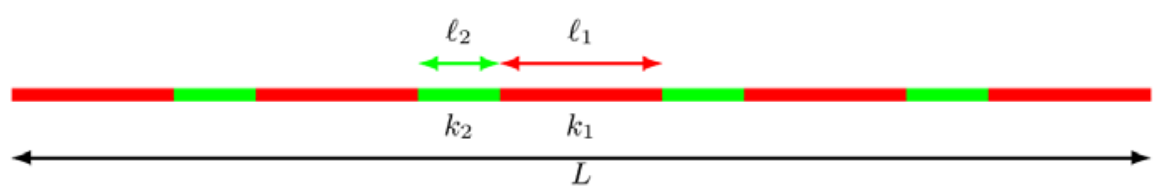

The bar being made up of two different media is heterogeneous, its equivalent rigidity is a periodic function $x$, where $x$ varies by taking the points of the bar. For asymptotic developments we will consider a function $k(y)$, set in $\mathbb{R}$. The period is divided into two parts, the first having the length $L_{1}=N_{c} \ell_{1}$ and the second $L_{2}=N_{c} \ell_{2}$ where the function $k(y)$ takes the respective values $k_{1}$ or $k_{2}$. The bar is reconstituted by grouping together the equally stiff mediums. One obtains a bar in two parts : that of rigidity $k_{1}$ and that of rigidity $k_{2}$. We define $k(y)$ as follows :

$$
k(y)= \begin{cases}k_{1} & 0<y<L_{1}, \\ k_{2} & L_{1}<y<L .\end{cases}
$$

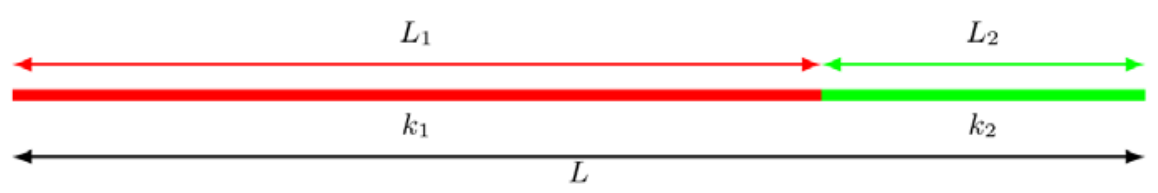

The bar has at point $x$ a stiffness which is $k\left(N_{c}\right)$. Indeed this function is periodic of period $\frac{L}{N_{c}}=\ell$ and it takes the value $k_{1}$ on the part of the length of the bar $\frac{L_{1}}{N_{c}}=\ell_{1}$ and $k_{2}$ on the part of the length of the bar $\frac{L_{2}}{N_{c}}=\ell_{2}$. Let us now consider several bars of the same length $L$, 
composed of a number $N_{c}$ of variable but large basic patterns. With a very large $N_{c}$, let's pose the small variable

$$
\varepsilon=\frac{1}{N_{c}}
$$

So we have $\ell=\varepsilon L, \ell_{1}=\varepsilon L_{1}$ and $\ell_{2}=\varepsilon L_{2}$ and the stiffness of the bar at point $x$ is $k\left(\frac{x}{\varepsilon}\right)$.

Remark 2. The bar stiffness also applies if the periodic stiffness of the bar is not constant in pieces. To do this we can simply change the definition (13) of the periodic function $k(y)$ on $\mathbb{R}$. The period of the function $k(y)$ is $[0 ; L]$ on $\mathbb{R}$.

Now let us take a bar fixed in $x=0$, subjected to a longitudinal force density $f$ and a force $F$ at point $x=L$.

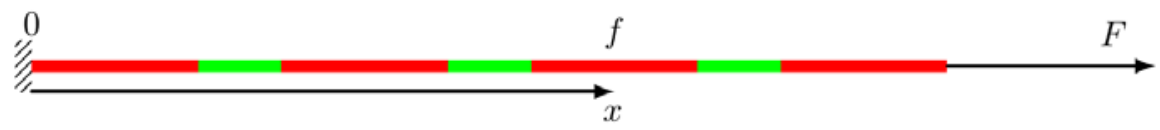

Figure 2. Loading of the bar.

\subsubsection{Equations of the problem}

Note $N$ the normal force in the bar, $u$ the points of the bar displacement. They check the following equations:

Equation of equilibrium:

$$
\frac{d N}{d x}+f=0
$$

Law of behaviour:

$$
N=k\left(\frac{x}{\varepsilon}\right) \frac{d u}{d x}
$$

Boundary conditions:

$$
u(0)=0 \quad \text { and } \quad N(L)=F .
$$




\subsubsection{Analytical solutions}

The problems (15), (16), (17) being one-dimensional, it is easy to determine the exact solution, in order to study the dependence of this solution on the number of patterns in the bar.

After an integration of Equation (15) taking into account the boundary conditions (17), we obtain

$$
N(x)=\int_{x}^{L} f(\xi) d \xi+F,
$$

by integrating this expression in (16), we have

$$
\frac{d u}{d x}=\frac{1}{k\left(\frac{x}{\varepsilon}\right)}\left(\int_{x}^{L} f(\xi) d \xi+F\right) .
$$

In a general case, we integrate this equation by using a primitive; in the considered case where the stiffness is constant in pieces on a segment $[a, b]$ we take for stiffness $k_{i}$. So we have

$$
u(x)=u(a)+\frac{1}{k_{i}}\left(P_{N}(x)-P_{N}(a)\right)
$$

where $P_{N}(x)$ is a primitive of $N$ on the segment $[a, b]$. In the segment $\left[0, \ell_{1}\right]$, we have $u\left(\ell_{1}\right)=\frac{1}{k_{1}}\left(P_{N}\left(\ell_{1}\right)-P_{N}(0)\right)$ which allows to determine $u$ on the segment $\left[\ell_{1}, \ell_{2}\right]$ and we continue step by step.

\subsubsection{Numerical examples}

Let us give values to the different geometrical and mechanical data:

$$
L=2 ; L_{1}=1.4 ; L_{2}=0.6 ; k_{1}=1.5 ; k_{2}=0.562 .
$$

Let us take $N_{c}=50, f=-\frac{\pi^{2}}{L^{2}} \sin \left(\frac{\pi}{L} x\right), F=-\frac{\pi}{L}$, by plotting on the same graph the movements of the heterogeneous bar and the homogeneous global stiffness bar $k^{G}$, we obtain the following figure: 


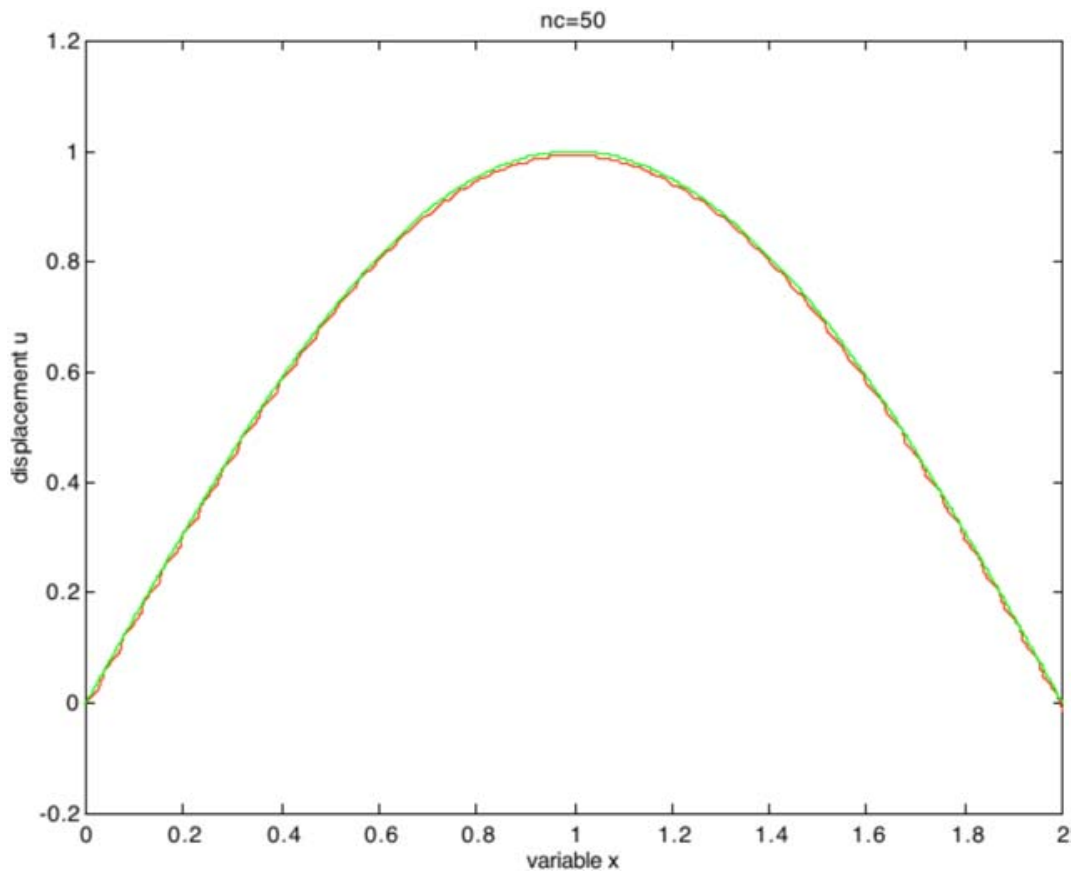

So, we notice " experimentally " that for $N_{c}$ rather large, the displacement graph is confused with that of a homogeneous bar of stiffness $k^{G}$.

\subsubsection{Heuristic method}

The previous result can be experimentally justified by the " heuristic " method introduced by Sanchez and developed by Suquet. To do this, the graphs in the $N_{c}$ ratio are magnified around the point $x=0.5$. 
Let us take $f=-\frac{\pi^{2}}{L^{2}} \sin \left(\frac{\pi}{L} x\right), F=-\frac{\pi}{L}$.

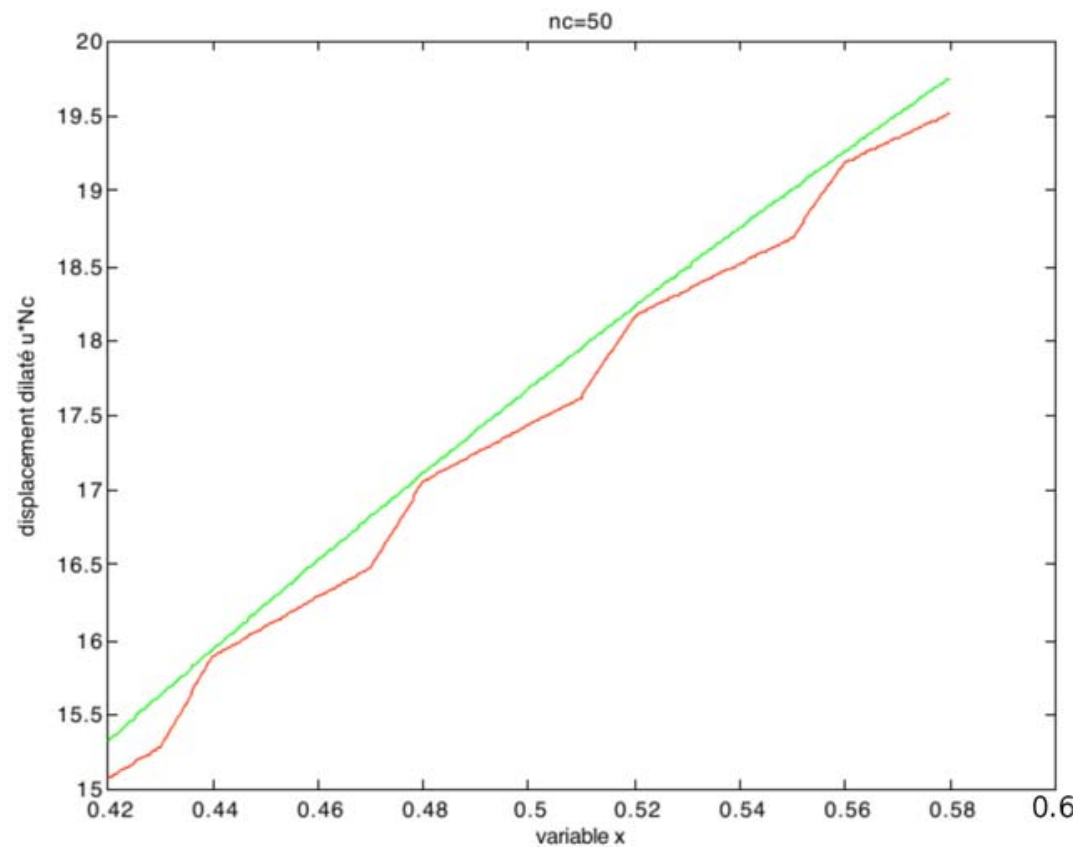

We can see that the variation in the exact displacement $u$ (in red) over a period of time $[x ; x+\ell]$ is practically the same as the limit displacement $U$ (in green), i.e.,

$$
u(x+\ell)-u(x) \simeq \ell \frac{d u}{d x} .
$$

For small $\ell$, the variation in normal effort over a period of time $[x ; x+\ell]$ is negligible. Therefore, the period over the normal effort $N$ is roughly constant. So the integration of the law of behaviour $\frac{d u}{d \xi}=\frac{N}{k\left(\frac{\xi}{\varepsilon}\right)}$ over a period of time $[x ; x+\ell]$ gives

$$
u(x+\ell)-u(x)=N \int_{x}^{x+\ell} \frac{1}{k\left(\frac{\xi}{\varepsilon}\right)} d \xi
$$


which gives the approximate condition of (19), results in

$$
N \int_{x}^{x+\ell} \frac{1}{k\left(\frac{\xi}{\varepsilon}\right)} d \xi=\ell \frac{d U}{d x}
$$

That is the law of macroscopic behaviour:

$$
N=k^{H} \frac{d U}{d x}
$$

where the homogenized stiffness $k^{H}$ is given by

$$
\frac{1}{k^{H}}=\frac{1}{\ell} \int_{x}^{x+\ell} \frac{1}{k\left(\frac{\xi}{\varepsilon}\right)} d \xi .
$$

By making a variable change and taking into account the periodicity of $k(y)$, the homogenized stiffness $k^{H}$ is independent of $x$.

$$
\frac{1}{k^{H}}=\frac{1}{L} \int_{0}^{L} \frac{1}{k(y)} d y
$$

In the case considered where the stiffness $k(y)$ is constant in pieces, it is easily verified that:

$$
\frac{L}{k^{H}}=\frac{L_{1}}{k_{1}}+\frac{L_{2}}{k_{2}}
$$

The stiffness $k^{H}$ is well equal to the overall stiffness $k^{G}$.

\subsubsection{Double scale development}

For a large number $N_{c}$, the displacement of the heterogeneous bar under a given load is close to that of a homogeneous bar under the same load. When $\varepsilon \rightarrow 0$ we can approximate the displacement of the heterogeneous bar by that of the homogeneous bar. " homogenize " is like stretching $\varepsilon \rightarrow 0$. 
The displacements as well as the voltages in these bars depend on the parameter $\varepsilon$ and are noted $u_{\varepsilon}$ and $N^{\varepsilon}$.

The determination of the limits for $\varepsilon \rightarrow 0$ of these quantities is an asymptotic study that can be done by constructing an asymptotic development of $u_{\varepsilon}$ and $N^{\varepsilon}$ with respect to $\varepsilon$.

Let us take $f=-\frac{\pi^{2}}{L^{2}} \sin \left(\frac{\pi}{L} x\right), F=-\frac{\pi}{L}$.

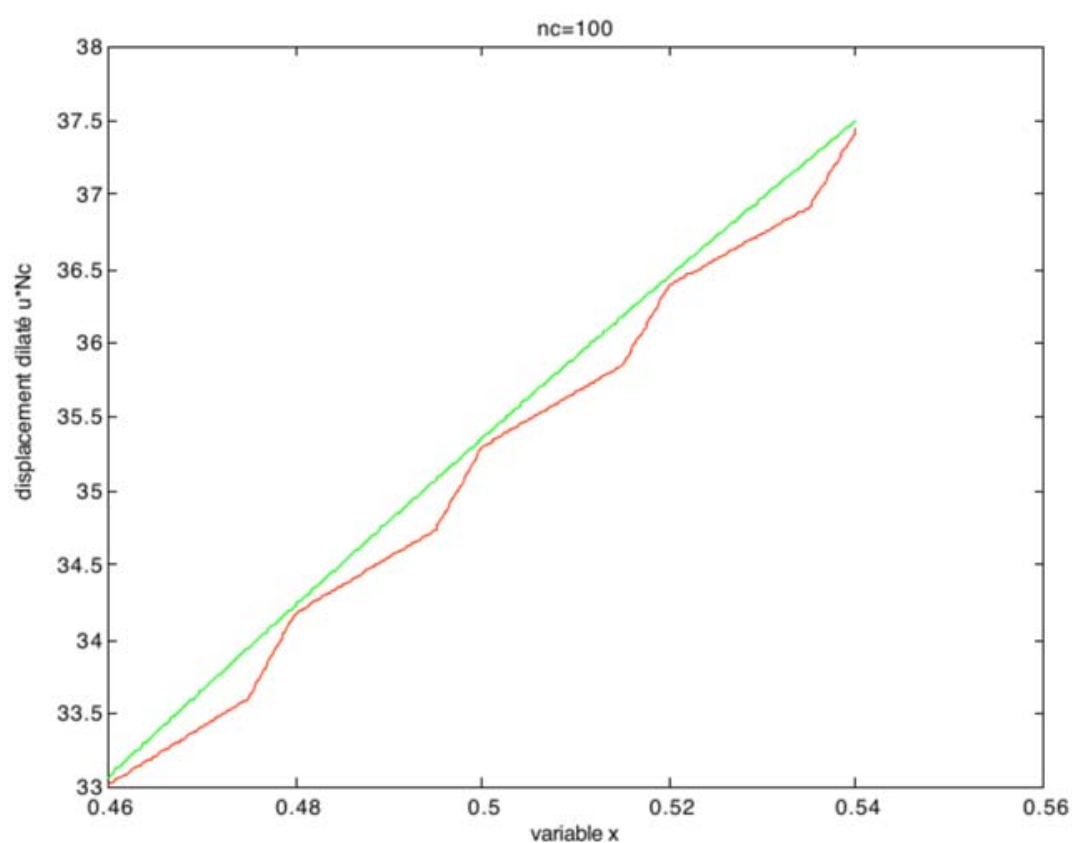

The difference between the exact solution $u$ and the limit solution $U$ is a perturbation which is in the order of $\frac{1}{N_{c}}=\varepsilon$ and this perturbation is, at least locally, periodic of period $\ell$. That can be written locally:

$$
u(x) \simeq U(x)+\varepsilon v(x)
$$

where $v$ is a locally periodic function of period $\ell$. 
This approximation is a beginning of development that is generalized and systematized in the form of

$$
u_{\varepsilon}(x)=u_{0}\left(x, \frac{x}{\varepsilon}\right)+\varepsilon u_{1}\left(x, \frac{x}{\varepsilon}\right)+\varepsilon^{2} u_{2}\left(x, \frac{x}{\varepsilon}\right)+\cdots,
$$

where $u_{i}(x, y)$ is a function $y$-periodic of period $Y$.

This development is called double scale development because it shows two scales of variation.

Indeed, a variation of $x$ in the order of $\varepsilon$ causes variations of $\varepsilon$ order of $u_{n}(x, y)$ relative to the first variable but of order 1 relative to the second. Variable $x$ is called a large scale variable and $y$ a small scale variable.

Let $\bar{x}$ and $\bar{x}+\varepsilon L$ be two points on the bar, according to the development (23), we have

$$
\begin{array}{ll}
u_{\varepsilon}(\bar{x}) & =u_{0}(\bar{x})+\varepsilon u_{1}\left(\bar{x}, \frac{\bar{x}}{\varepsilon}\right)+\varepsilon^{2}(\cdots) \\
u_{\varepsilon}(\bar{x}+\varepsilon L) & =u_{0}(\bar{x}+\varepsilon L)+\varepsilon u_{1}\left(\bar{x}+\varepsilon L, \frac{\bar{x}}{\varepsilon}+L\right)+\varepsilon^{2}(\cdots) .
\end{array}
$$

Let us consider the periodicity of the functions $u_{n}$ and using Taylor developments:

$$
u_{\varepsilon}(\bar{x}+\varepsilon L)=u_{0}(\bar{x})+\varepsilon\left(u_{1}\left(\bar{x}, \frac{\bar{x}}{\varepsilon}\right)+\frac{d u_{0}}{d x}(\bar{x}) L\right)+\varepsilon^{2}(\cdots)
$$

and by difference:

$$
u_{\varepsilon}(\bar{x}+\varepsilon L)-u_{0}(\bar{x})=\varepsilon \frac{d u_{0}}{d x}(\bar{x}) L+\varepsilon^{2}(\cdots)
$$

We find, at order $\varepsilon^{2}$ near equality:

$$
u_{\varepsilon}(\bar{x}+\varepsilon L)-u_{0}(\bar{x})=\varepsilon \frac{d u_{0}}{d x}(\bar{x}) L,
$$

that was used in the heuristic method. 
By derivation of (23), it comes

$$
\begin{aligned}
\frac{d u_{\varepsilon}}{d x}(x)=\frac{1}{\varepsilon} \frac{d u_{0}}{d y}\left(x, \frac{x}{\varepsilon}\right)+\frac{d u_{0}}{d x}\left(x, \frac{x}{\varepsilon}\right) & +\frac{d u_{1}}{d y}\left(x, \frac{x}{\varepsilon}\right) \\
& +\varepsilon\left(\frac{d u_{1}}{d x}\left(x, \frac{x}{\varepsilon}\right)+\frac{d u_{2}}{d y}\left(x, \frac{x}{\varepsilon}\right)\right)+\cdots,
\end{aligned}
$$

which reported in the law of behaviour (16), shows that the normal force $N_{\varepsilon}$ develops in

$$
N_{\varepsilon}(x)=\frac{1}{\varepsilon} N^{(-1)}\left(x, \frac{x}{\varepsilon}\right)+N^{(0)}\left(x, \frac{x}{\varepsilon}\right)+\varepsilon N^{(1)}\left(x, \frac{x}{\varepsilon}\right)+\varepsilon^{2} N\left(x, \frac{x}{\varepsilon}\right)+\cdots,
$$

where the $N^{(n)}(x, y)$ are $y$-periodic of period $Y$ and verify by identification terms of the same power of $\varepsilon$ :

$$
\begin{aligned}
& N^{(-1)}=k(y) \frac{d u_{0}}{d y}, \\
& N^{(0)}=k(y)\left(\frac{d u_{0}}{d x}+\frac{d u_{1}}{d y}\right), \\
& N^{(1)}=k(y)\left(\frac{d u_{1}}{d x}+\frac{d u_{2}}{d y}\right),
\end{aligned}
$$

By derivation, we obtain by omitting the variables:

$$
\begin{aligned}
\frac{d N_{\varepsilon}}{d x}=\frac{1}{\varepsilon^{2}} \frac{d N^{(-1)}}{d y} & +\frac{1}{\varepsilon}\left(\frac{d N^{(-1)}}{d x}+\frac{d N^{(0)}}{d y}\right) \\
& +\frac{d N^{(0)}}{d x}+\frac{d N^{(1)}}{d y}+\varepsilon\left(\frac{d N^{(1)}}{d x}+\frac{d N^{(2)}}{d y}\right)+\cdots .
\end{aligned}
$$


The transfer of this development into the equation of equilibrium (15) gives after identification of terms of the same powers of $\varepsilon$

$$
\begin{array}{r}
\frac{d N^{(-1)}}{d y}=0, \\
\frac{d N^{(-1)}}{d x}+\frac{d N^{(0)}}{d y}=0, \\
\frac{d N^{(0)}}{d x}+\frac{d N^{(1)}}{d y}+f=0,
\end{array}
$$

From Equation (25a) we deduce that $N^{(-1)}$ does not depend on $y$ (which is obviously compatible with the periodicity in $y$ ). Equation (24a) is then integrated in

$$
u_{0}(x, y)=N^{(-1)}(x) \int_{0}^{y} \frac{1}{k(\zeta)} d \zeta+\tilde{u}_{0}(x)
$$

where $\tilde{u}_{0}(x)$ is an integration constant for the differential equation in $y$ (25a), i.e., a function of the large-scale variable $x$ alone.

The periodicity condition $u_{0}(x, L)=u_{0}(x, 0)$ is equivalent to:

$$
N^{(-1)} \int_{0}^{L} \frac{1}{k(\zeta)} d \zeta=0
$$

$k$ being strictly positive, we have

$$
N^{(-1)}=0,
$$

and

$$
u_{0}(x, y)=\tilde{u}_{0}(x)
$$

i.e., $u_{0}$ depends only on the large-scale variable $x$. 
To obtain the «macroscopic» equations satisfied by $u_{0}$, we use Equations (25) and (24).

$N^{(-1)}$ being zero, Equation (25b) implies that $N^{(0)}$ does not depend on $y$. The integration on $[0 ; L]$ of Equation (24b) rewritten:

$$
\frac{d u_{0}}{d x}+\frac{d u_{1}}{d y}=\frac{1}{k(y)} N^{(0)}
$$

then gives, taking into account the periodicity of $u_{1}$ and the independence of $u_{0}$ with respect to $y$ :

$$
L \frac{d u_{0}}{d x}=N^{(0)} \int_{0}^{L} \frac{1}{k(\zeta)} d \zeta
$$

That we can write

$$
N^{(0)}=k^{H} \frac{d u_{0}}{d x},
$$

with in the case considered where $k$ is constant in pieces

$$
\frac{L}{k^{H}}=\frac{L_{1}}{k_{1}}+\frac{L_{2}}{k_{2}} \text {. }
$$

This law of macroscopic behaviour is exactly the law (20) found by the heuristic method.

To determine the equilibrium equation verified by $N^{(0)}$, we integrate Equation (25c) on $[0 ; L]$, which gives, considering the periodicity of $N^{(1)}$ :

$$
\frac{d N^{(0)}}{d x}+\langle f\rangle=0
$$

where the average $\langle f\rangle$ of $f$ is defined by

$$
\langle f\rangle=\frac{1}{L} \int_{0}^{L} f(y) d y .
$$

The asymptotic method thus made it possible to determine the macroscopic equivalent model of the finely heterogeneous bar constituted of the homogenized constitutive law (27) and the macroscopic equilibrium equation (28). 


\subsubsection{Convergence}

The results of the asymptotic double scale developments of the previous paragraph remain formal until such time as convergence has not been proven. Several convergence methods have been applied to the homogenization of periodic media, $G$-convergence, $\Gamma$-convergence, $H$-convergence, the so-called " Tartar " method, two-scale convergence, bursting method. The two-scale convergence method will be studied in the next section.

\section{Two-Scale Convergence}

This section is devoted to the concept of two-scale convergence and some of its properties. We highlight how some results regarding two-scale convergence relate to traditional results of weak and strong convergence.

Weak convergence does not keep information on the local behaviour of functions. This loss of information in weak limit causes some "unpleasant" properties. The study of the function $\sin \left(\frac{2 \pi x}{\varepsilon}\right)$ is a particular example.
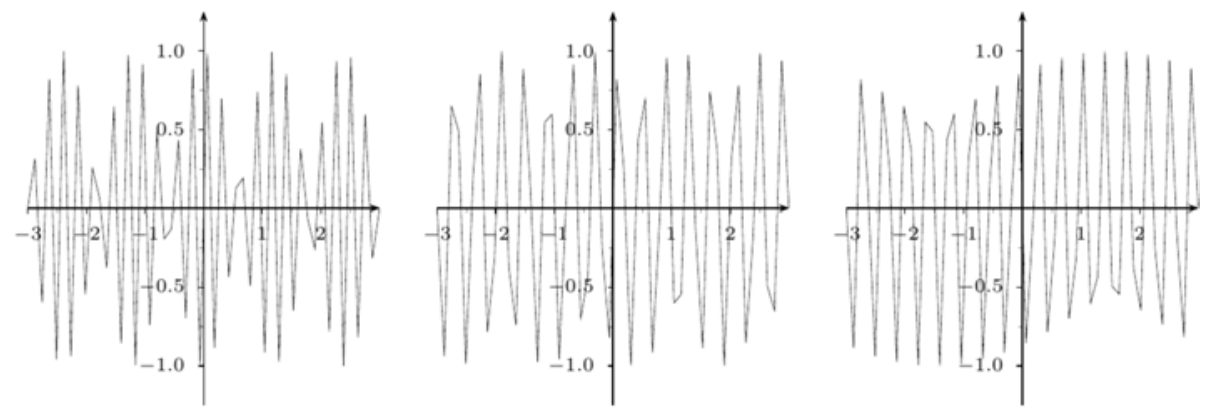

Figure 3. Graph of $\sin \left(\frac{2 \pi x}{\varepsilon}\right)$ with $\varepsilon=\frac{1}{20}, \frac{1}{120}, \frac{1}{520}$. 
If we take $u_{\varepsilon}(x)=v_{\varepsilon}(x)=\sin \left(\frac{2 \pi x}{\varepsilon}\right), u_{\varepsilon}$ and $v_{\varepsilon}$ converge weakly in $L^{2}(0, \pi)$ to 0 . But the product $u_{\varepsilon} v_{\varepsilon}$ converges to $\frac{1}{2}$. The previous example shows that the evolution of a problem is linked to small perturbations. These perturbations cannot be neglected because they can lead to completely divergent results in reality.

The two-scale convergence allows to capture the oscillations of a function in reasoning with that of the type $\varphi\left(x, \frac{x}{\varepsilon}\right)$, a periodic test function. In some cases, therefore, two-scale convergence makes it possible to overcome the loss of information on local behaviour, it is an intermediate convergence between weak convergence and strong convergence. The test function $\varphi\left(x, \frac{x}{\varepsilon}\right)$ being a function of one variable becomes by passing to the limit two-scale, a function $\varphi(x, y)$ dependent on two variables. The variable $y=\frac{x}{\varepsilon} \in Y$ is the fast variable also called microscopic variable, while $x \in \Omega$ is the slow variable also called macroscopic variable.

In this section, we will give first the essential definitions of two-scale convergence in $L^{P}$. Secondly, we will give some homogenization problems solved by two-scale convergence. And we will finish by an application of two-scale convergence to a homogenization problem.

\subsection{Definition and essential properties of two-scale convergence in $L^{p}$}

In this part, we give the definition of two-scale convergence as well as some properties and examples related to two-scale convergence.

The idea of two-scale convergence consists in moving from a sequence of one variable functions to two variables. 
We pose

$$
\|w\|_{p}=\left(\int_{Y}|w(y)|^{p} d y\right)^{\frac{1}{p}}, \quad w \in L_{\sharp}^{p}(Y) .
$$

We define a norm in $L_{\sharp}^{p}(Y)$ which in fact is a Banach space (a Hilbert if $p=2)$.

\subsubsection{Definition of two-scale convergence}

For all function $\psi \in L^{p}\left(\Omega ; C_{\sharp}(Y)\right)$, we designate by $\psi^{\varepsilon}$ the function of $L^{p}(\Omega)$ defined by

$$
\psi^{\varepsilon}(x)=\psi\left(x, \frac{x}{\varepsilon}\right), \quad x \in \Omega, \quad \varepsilon>0 .
$$

We give a fundamental result.

Lemma 2 ([55], p. 1). When $\varepsilon \rightarrow 0$, we have $\psi^{\varepsilon} \rightarrow \widetilde{\phi}$ in $L^{p}(\Omega)$-weak, where $\widetilde{\psi}(x)=\int_{Y} \psi(x, y) d y$ is a $L^{p}(\Omega)$ function.

In other words:

$$
\begin{array}{r}
\lim _{\varepsilon \rightarrow 0} \int_{\Omega} \psi^{\varepsilon}(x) \varphi(x) d x=\int_{\Omega} \widetilde{\psi}(x) \varphi(x) d x=\int_{\Omega} \int_{Y} \psi(x, y) \varphi(x) d y d x, \quad \forall \varphi \in L^{q}(\Omega), \\
\text { where } \frac{1}{p}+\frac{1}{q}=1 .
\end{array}
$$

Let's define now the notion of two-scale convergence.

Definition 1. A sequence (generalize) $\left(u_{\varepsilon}\right)_{\varepsilon>0}$ of functions in $L^{p}(\Omega)$ is said two-scale convergent in $L^{p}(\Omega)$ to a function $u_{0} \in L^{p}(\Omega \times Y)$, if for $\varepsilon \rightarrow 0$, we have

$$
\int_{\Omega} u_{\varepsilon}(x) \psi\left(x, \frac{x}{\varepsilon}\right) d x \rightarrow \int_{\Omega} \int_{Y} u_{0}(x, y) \psi(x, y) d y d x, \quad \forall \psi \in L^{q}\left(\Omega ; C_{\sharp}(Y)\right) .
$$


Remark 3. Nguetseng has also extended two-scale convergence beyond periodicity, see [56].

Remark 4 ([53], p. 17). $u_{\varepsilon}$ means $u_{\varepsilon_{n}}$ with $\varepsilon_{n} \underset{n \rightarrow+\infty}{\longrightarrow} 0$. The $n$ index will be omitted routinely.

Remark 5 ([49], p. 17). There is a confusion in literature about the choice of the space of the test function. Some authors take the test function in $\mathcal{D}\left(\Omega ; C_{\sharp}^{\infty}(Y)\right)$ instead of $L^{p}(\Omega)$.

In the Example 4 ([49], p. 11), the authors give an example of a sequence $\left(u_{\varepsilon}\right)$ in $L^{p}(\Omega)$ satisfying (30) for $\psi \in \mathcal{D}\left(\Omega ; C_{\sharp}^{\infty}(Y)\right)$ but which is neither bounded nor weakly convergent in $L^{p}(\Omega)$.

In the Example 5 ([49], p. 11), they replace $\mathcal{D}\left(\Omega ; C_{\sharp}^{\infty}(Y)\right)$ by $C\left(\Omega ; C_{\sharp}^{\infty}(Y)\right)$ and they give an example of test function $\psi \in C\left(\Omega ; C_{\sharp}^{\infty}(Y)\right)$, for which the sequence $\left(u_{\varepsilon}\right)$ does not converge weakly in $L^{p}(\Omega)$ to the function $v(x)$ defined by $\int_{Y} u(x, y) d y$. This sequence is not bounded also.

Then, when $\left(u_{\varepsilon}\right)$ is bounded in $L^{p}(\Omega)$ we can take $\psi(x, y)$ in $\mathcal{D}\left(\Omega ; C_{\sharp}^{\infty}(Y)\right)$.

Proposition 1 ([49], p. 12). Let $\left(u_{\varepsilon}\right)$ be a bounded sequence in $L^{p}(\Omega)$ such that

$$
\int_{\Omega} u_{\varepsilon}(x) \varphi\left(x, \frac{x}{\varepsilon}\right) d x \rightarrow \int_{\Omega} \int_{Y} u(x, y) \psi(x, y) d y d x
$$

for every $\psi \in \mathcal{D}\left(\Omega ; C_{\sharp}^{\infty}(Y)\right)$. Then $u_{\varepsilon}$ two-scale converges to $u$. 
Proposition 2 ([49], p. 6). Let's be $\psi$ a continuous function from $\mathbb{R}_{x}^{N} \times \mathbb{R}_{y}^{N}$ to $\mathbb{R}$. We suppose again that for all $x=\left(x_{1}, x_{2}, \ldots, x_{N}\right)$, the partial function $y \rightarrow \psi(x, y)$ from $\mathbb{R}_{y}^{N}$ to $\mathbb{R}$ is Y-periodic. Then, we suppose that $\Omega$ is bounded, and we pose

$$
u_{\varepsilon}(x)=\psi\left(x, \frac{x}{\varepsilon}\right), \quad x \in \Omega,
$$

for all $\varepsilon>0$. The sequence $\left(u_{\varepsilon}\right)_{\varepsilon>0}$ is weakly two-scale convergent in $L^{p}(\Omega)$ to the function $u_{0} \in L^{p}\left(\Omega ; L_{\sharp}^{p}(Y)\right)$ defined by

$$
u_{0}(x, y)=\psi(x, y), \quad x \in \Omega, y \in \mathbb{R}_{y}^{N} .
$$

Proof. Let's show that

$$
\lim _{\varepsilon \rightarrow 0} \int_{\Omega} u_{\varepsilon}(x) \varphi\left(x, \frac{x}{\varepsilon}\right) d x=\int_{\Omega} \int_{Y} u_{0}(x, y) \varphi(x, y) d y d x \forall \varphi \in L^{q}\left(\Omega, C_{\sharp}(Y)\right),
$$

i.e.,

$$
\lim _{\varepsilon \rightarrow 0} \int_{\Omega} \psi\left(x, \frac{x}{\varepsilon}\right) \varphi\left(x, \frac{x}{\varepsilon}\right) d x=\int_{\Omega} \int_{Y} \psi(x, y) \varphi(x, y) d y d x
$$

Let's pose that $\eta^{\varepsilon}(x)=\psi\left(x, \frac{x}{\varepsilon}\right) \varphi\left(x, \frac{x}{\varepsilon}\right)$.

Obviously we have $u_{\varepsilon} \in L^{p}(\Omega)$, because $u_{\varepsilon}$ is continuous and $\Omega$ is bounded.

We pose $v_{\varepsilon}(x)=\varphi\left(x, \frac{x}{\varepsilon}\right)$.

$v_{\varepsilon} \in L^{p}(\Omega)$ because $\Omega$ is bounded. Then $\eta^{\varepsilon}(x) \in L^{p}(\Omega)$.

According to the Lemma 2, we have $\eta^{\varepsilon}(x) \rightarrow \eta(x)$ in $L^{p}(\Omega)$-weak where $\eta(x)=\int_{Y} \psi(x, y) \varphi(x, y) d y$. In other words, we have:

$$
\int_{\Omega} \eta^{\varepsilon}(x) \alpha(x) d x \rightarrow \int_{\Omega} \int_{Y} \psi(x, y) \varphi(x, y) \alpha(x) d y d x \quad \forall \alpha \in L^{q}(\Omega) .
$$


In particular $\alpha(x)=1$ ( $\Omega$ being bounded) leads to

$$
\lim _{\varepsilon \rightarrow 0} \int_{\Omega} \psi\left(x, \frac{x}{\varepsilon}\right) \varphi\left(x, \frac{x}{\varepsilon}\right) d x=\int_{\Omega} \int_{Y} \psi(x, y) \varphi(x, y) d y d x .
$$

Proposition 3 ([G. Nguetseng]). Let's be $\psi$ the function from $\mathbb{R}_{x}^{N} \times \mathbb{R}_{y}^{N}$ to $\mathbb{R}$ given by

$$
\psi(x, y)=\varphi(x) u(y), \quad x \in \mathbb{R}^{N}, y \in \mathbb{R}^{N},
$$

where $\varphi$ is continuous with compact support in $\mathbb{R}_{x}^{N}$, and $u \in C_{\sharp}(Y)$.

We define $u_{\varepsilon}$ like in (31), where $\Omega$ isn't supposed bounded.

Then, the sequence $\left(u_{\varepsilon}\right)_{\varepsilon>0}$ weakly two-scale converges in $L^{p}(\Omega)$ to the function $u_{0}$ given by (32).

Proof. Let's show that $\int_{\Omega} \varphi(x) u(x / \varepsilon) h(x, x / \varepsilon) d x \rightarrow \int_{\Omega} \int_{Y} \varphi(x) u(y)$ $h(x, y) d y d x, \quad \forall h \in L^{q}(\Omega \times Y) \mathcal{D}(\Omega \times Y) \quad$ is dense in $L^{q}(\Omega \times Y)$ so it comes down to showing that

$$
\int_{\Omega} \varphi(x) u(x / \varepsilon) h(x, x / \varepsilon) d x \rightarrow \int_{\Omega} \int_{Y} \varphi(x) u(y) h(x, y) d y d x, \quad \forall h \in \mathcal{D}(\Omega \times Y),
$$

$\mathcal{D}(\Omega) \otimes \mathcal{D}(Y)$ is dense in $\mathcal{D}(\Omega \times Y)$ so it comes down to showing that

$$
\begin{gathered}
\int_{\Omega} \varphi(x) u(x / \varepsilon) h_{1}(x) h_{2}(x / \varepsilon) d x \rightarrow \int_{\Omega} \int_{Y} \varphi(x) u(y) h_{1}(x) h_{2}(y) d y d x, \quad \forall h_{1} \in \mathcal{D}(\Omega), \\
\forall h_{2} \in \mathcal{D}(Y) \\
\int_{\Omega} \varphi(x) u(x / \varepsilon) h_{1}(x) h_{2}(x / \varepsilon) d x=\int_{\Omega} \varphi(x) u(x / \varepsilon) h_{2}(x / \varepsilon) h_{1}(x) d x
\end{gathered}
$$


Let's pose $v(x / \varepsilon)=u(x / \varepsilon) h_{2}(x / \varepsilon)$ we have $\int_{\Omega} \varphi(x) u(x / \varepsilon) h_{2}(x / \varepsilon) h_{1}(x) d x$ $=\int_{\Omega} \varphi(x) v(x / \varepsilon) h_{1}(x) d x . \varphi$ is continuous with compact support and $v(x / \varepsilon) \in C_{\sharp}(Y)$ then $\varphi(x) v(x / \varepsilon)$ is continuous with compact support.

According to the Lemma $2, \int_{\Omega} \varphi(x) v(x / \varepsilon) h_{1}(x) d x \rightarrow \int_{\Omega} \int_{Y} \varphi(x) v(y) h_{1}$ $(x) d y d x$.

We conclude that $\int_{\Omega} u_{\varepsilon}(x) h(x, x / \varepsilon) d x \rightarrow \int_{\Omega} \int_{Y} u_{0}(x, y) h(x, y) d y d x, \forall h \in L^{q}(\Omega \times Y)$, where $u_{0}(x, y)=\psi(x, y)=\varphi(x) v(y)$.

Proposition 4. Let's be $\psi$ the function from $\mathbb{R}_{x}^{N} \times \mathbb{R}_{y}^{N}$ to $\mathbb{R}$ given by

$$
\psi(x, y)=\varphi(x) u(y), \quad x \in \Omega \subset \mathbb{R}^{N}, y \in \mathbb{R}^{N},
$$

where $\varphi$ is bounded with compact support in $\mathbb{R}_{x}^{N}$, and $u \in C_{\sharp}(Y)$.

We define $u_{\varepsilon}$ like in (31), where $\Omega$ isn't supposed bounded.

Then, the sequence $\left(u_{\varepsilon}\right)_{\varepsilon>0}$ weakly two-scale converges in $L^{p}(\Omega \times Y)$ to the function $u_{0}$ given by (32).

Proof. Let's show that

$$
\lim _{\varepsilon \rightarrow 0} \int_{\Omega} u_{\varepsilon}(x) h(x, x / \varepsilon) d x=\int_{\Omega} \int_{Y} u_{0}(x, y) h(x, y) d y d x h \in L^{q}(\Omega \times Y) .
$$

We have

$$
\lim _{\varepsilon \rightarrow 0} \int_{\Omega} u_{\varepsilon}(x) h(x, x / \varepsilon) d x=\lim _{\varepsilon \rightarrow 0} \int_{\Omega} \varphi(x) u(x / \varepsilon) h(x, x / \varepsilon) d x .
$$


$\varphi$ is bounded with compact support and $u$ is continuous $\Rightarrow \varphi \otimes u$ is bounded then $\varphi \otimes u \in L^{\infty}(\Omega)$. As $\varphi \otimes u$ is with compact support then $\varphi \otimes u \in L^{r}(\Omega) \forall r$.

It is sufficient that $\frac{1}{r}=\frac{1}{p}-\frac{1}{q}$ so that $u_{\varepsilon}(x)=\varphi(x) u(x / \varepsilon) \in L^{p}(\Omega)$.

According to the Lemma 2,

$$
\begin{aligned}
\lim _{\varepsilon \rightarrow 0} \int_{\Omega} \varphi(x) u(x / \varepsilon) h(x, x / \varepsilon) d x & =\int_{\Omega} \int_{Y} \varphi(x) u(y) h(x, y) d y d x \\
& =\int_{\Omega} \int_{Y} u_{0}(x, y) h(x, y) d y d x
\end{aligned}
$$

Now we will give some easy examples of sequences which two-scale converges.

\subsubsection{Examples}

The notation is the same like the above.

Example 1. Let's take $N=1,1 \leqslant p<+\infty, \Omega=[0,1[$ and $Y=[0 ; 2 \pi]$.

We pose $\psi(x, y)=\sin (2 \pi y), u_{\varepsilon}(x)=\sin (2 \pi x / \varepsilon), u_{\varepsilon}$ is continuous on $\Omega$ and $\Omega$ is bounded then $u_{\varepsilon} \in L^{p}(\Omega)$.

$y \rightarrow \psi(x, y)=\sin (2 \pi y)$ is $Y$-periodic.

According to the Proposition 2, we deduce that $\sin (2 \pi x / \varepsilon)$ converges two-scale in $L^{p}(\Omega)$ to $\sin (2 \pi y) \in L^{p}\left(\Omega ; L_{\sharp}^{p}(Y)\right)$.

Example 2. Let's take $N=1,1 \leqslant p<+\infty, \Omega=\mathbb{R}$ and $Y=[0 ; 1]$.

We pose $\psi(x, y)=\varphi(x) u(y)$ with $\varphi(x)=2(x)$ if $x \in[0,1 / 2[,-2 x+2$ if $x \in[1 / 2 ; 1[$ and 0 elsewhere, we take $u(y)=\sin (2 \pi y)$.

$\varphi$ is continuous with compact support in $\mathbb{R}_{x}$. 
$u(y)$ is continuous and $Y$-periodic.

We define $u_{\varepsilon}(x)=\psi(x, x / \varepsilon), \forall x \in \Omega$.

According to the Proposition 3, we deduce that $\varphi(x) \sin (2 \pi x / \varepsilon)$ converges two-scale in $L^{p}(\Omega)$ to $\varphi(x) \sin (2 \pi y) \in L^{p}\left(\Omega ; L_{\sharp}^{p}(Y)\right)$.

Example 3. If we take $u_{\varepsilon}(x)=\frac{1}{2} \sin \left(\frac{x}{\varepsilon}\right)+\frac{1}{2} \sin (x)+1$ with $b_{1}(x)=\frac{1}{2}$, $a(x / \varepsilon)=\sin \left(\frac{x}{\varepsilon}\right)$ and $b_{2}(x)=\frac{1}{2} \sin (x)+1 . a(x / \varepsilon)$ converges weakly to 0 , then $u_{\varepsilon}(x)$ converges weakly to $\frac{1}{2} \sin (x)+1$ and it two-scale converges (strongly) to $\frac{1}{2} \sin (y)+\frac{1}{2} \sin (x)+1$.

Remark 6. To better understand this example, see properties (4) and (5) on the next page.

\subsubsection{General properties}

Theorem 1 ([49], p. 6). Let $f(x, y) \in L^{1}\left(\Omega, C_{\sharp}(Y)\right)$. Then $f\left(x, \frac{x}{\varepsilon}\right)$ is a measurable function on $\Omega$ such that

$$
\left\|f\left(x, \frac{x}{\varepsilon}\right)\right\|_{L^{1}(\Omega)} \leqslant\|f(x, y)\|_{L^{1}\left(\Omega, C_{\sharp}(Y)\right)}=\int_{\Omega} \sup _{y \in Y}|f(x, y)| d x,
$$

and

$$
\lim _{\varepsilon \rightarrow 0} \int_{\Omega} f\left(x, \frac{x}{\varepsilon}\right) d x=\int_{\Omega} \int_{Y} f(x, y) d y d x
$$

Proof. For the proof, see ([49], pp. 6-7).

We now expose a result of compactness for two-scale convergence.

Theorem 2 ([49], p. 13). From any bounded sequence $u_{\varepsilon}$ of $L^{p}(\Omega)$, we can extract a subsequence that converges two-scale to a limit $u_{0}(x, y) \in L^{p}(\Omega \times Y)$. 
Proof. For the proof, see ([49], p. 13-14).

Simple cases of two-scale convergence (see [5], pp. 15-16) for properties (1) to (3) and ([53], p. 107-108) for properties (4) to (7).

(1) Any sequence $u_{\varepsilon}$ which converges strongly in $L^{2}(\Omega)$ to a limit $u(x)$, two-scale converges to the same limit $u(x)$.

(2) For any smooth function $u_{0}(x, y)$, being $Y$-periodic in $y$, the associated sequence $u_{\varepsilon}(x)=u_{0}\left(x, \frac{x}{\varepsilon}\right)$ two-scale converges to $u_{0}(x, y)$.

(3) Any sequence $u_{\varepsilon}$ which admits an asymptotic expansion of type $u_{\varepsilon}(x)=u_{0}\left(x, \frac{x}{\varepsilon}\right)+\varepsilon u_{1}\left(x, \frac{x}{\varepsilon}\right)+\varepsilon^{2} u_{2}\left(x, \frac{x}{\varepsilon}\right)+\cdots ;$ where the functions $u_{i}(x, y)$ are smooth and $Y$-periodic in $y$, converges two-scale to the first term of the expansion, namely $u_{0}(x, y)$ since the Theorem 1 implies that $\int_{\Omega} u_{i}\left(x, \frac{x}{\varepsilon}\right) \psi\left(x, \frac{x}{\varepsilon}\right) d x \rightarrow \int_{\Omega} \int_{Y} u_{0}(x, y) \psi(x, y) d y d x$ for all $\psi \in L^{p}\left(\Omega, C_{\sharp}(Y)\right)$.

(4) Let $a(y)$ be a $Y$-periodic bounded function with zero mean value $\int_{Y} a(y) d y=0$ and let $b_{1}(x), b_{2}(x) \in L^{p}(\Omega)$. Then, the sequence $\left(u_{\varepsilon}\right)$ defined by $u_{\varepsilon}(x)=b_{1}(x) a(x / \varepsilon)+b_{2}(x)$ converges weakly to $b_{2}(x)$ and it two-scale converges (strongly) to $b_{1}(x) a(y)+b_{2}(x)$. We see that the weak limit is the function $b_{2}$ only.

(5) Let us consider the same functions $a(y), b_{1}(x), b_{2}(x)$, but another sequence $\left(v_{\varepsilon}\right)$ defined by $v_{\varepsilon}(x)=b_{1}(x) a\left(x / \varepsilon^{2}\right)+b_{2}(x)$. Then the twoscale and weak limits coincide, which means that the two-scale limit is constant in the variable $y$. In this case, the information on oscillations is not kept. Similarly, taking a sequence given by $w_{\varepsilon}(x)=b_{1}(x) a(c x \varepsilon)+b_{2}$ with $c$ irrational, the two-scale limit equals to $b_{2}$ only. It is the consequence that diminishing the periods in sequences is not in resonance with the periods in the test function. 
(6) Let $u(y)$ be a $Y$-periodic function, we consider the functions $u_{0}(x, y)=u(y)$ and $\tilde{u}_{0}(y)=u(y-1 / 2)$. We define the sequence $\left(u_{\varepsilon}\right)$ by $u_{\varepsilon}(x)=\tilde{u}_{0}(x / \varepsilon)$.

Then $\left\|u_{\varepsilon}\right\|_{L^{p}(\Omega)} \rightarrow\left\|u_{0}\right\|_{L^{p}(\Omega \times Y)}$, but $\left(u_{\varepsilon}\right)$ two-scale converges to $\tilde{u}_{0}$.

(7) Let us consider sequences $\left(u_{\varepsilon}\right)$ from (4) and $\left(v_{\varepsilon}\right)$ from (5). Denoting the two-scale limit by $u_{0}$ and the weak limit by $u$, we have $\lim \left\|u_{\varepsilon}\right\|_{L^{p}(\Omega)}$ $=\left\|u_{0}\right\|_{L^{p}(\Omega \times Y)}>\|u\|_{L^{p}(\Omega)} ; \lim \left\|v_{\varepsilon}\right\|_{L^{p}(\Omega)}=\|u\|_{L^{p}(\Omega \times Y)}>\|u\|_{L^{p}(\Omega)}$ and finally the sum $u_{\varepsilon}+v_{\varepsilon}$ satisfies sharp inequalities.

Proposition 5 ([49], pp. 9-10). Let $u(x, y)$ be a smooth function defined on $\Omega \times \mathbb{R}^{N}, \quad Y$-periodic in $y$. Then the limit two-scale of $u_{\varepsilon}(x)=u\left(x, \frac{x}{\varepsilon^{2}}\right)$ is equal to the weak $L^{p}$ limit, namely $\int_{Z} u(x, z) d z$ where $Z$ is the unit cube in $\mathbb{R}^{N}$.

Proof. Indeed, let $f(x, y, z)$ be a smooth function which is $Y$-periodic in $y$ and $Z$-periodic in $z$. Then it is well known that

$$
f\left(x, \frac{x}{\varepsilon}, \frac{x}{\varepsilon^{2}}\right) \rightarrow \int_{Y} \int_{Z} f(x, y, z) d z d y \quad \text { weakly in } L^{p} .
$$

This implies that

$$
\int_{\Omega} u\left(x, \frac{x}{\varepsilon^{2}}\right) \phi\left(x, \frac{x}{\varepsilon}\right) d x \rightarrow \int_{\Omega} \int_{Y} \int_{Z} u(x, z) d z \phi(x, y) d y d x, \quad \forall \phi \in L^{q}\left(\Omega, C_{\sharp}(Y)\right) .
$$

In other words, the two-scale limit $v$ of $u_{\varepsilon}$ is $v(x)=\int_{Z} u(x, z) d z$.

As we can see, the previous compactness theorem generalizes the theorem in $L^{2}(\Omega)$ (from any bounded sequence in $L^{2}(\Omega)$ one can extract a subsequence which converges weakly in $L^{2}(\Omega)$ ). This is all the more 
not surprising since the definition of two-scale convergence is written under conditions of weak convergence. It is a convergence for any test function and not a standard convergence.

Two-scale convergence also allows us to justify convergence of the sequence of gradients $\nabla u_{\varepsilon}$ in the sense of the following proposal.

Proposition 6 ([41], p. 34). Let $u_{\varepsilon}$ be a bounded sequence in $H^{1}(\Omega)$. Then $u_{\varepsilon}$ converges two-scale, to a function $u_{0}(x) \in H^{1}(\Omega)$ and there is a function $u_{1}(x, y) \in L^{2}\left(\Omega ; H_{\sharp}^{1}(Y)\right)$ such that $\nabla u_{\varepsilon}$ two-scale converges to $\nabla_{x} u_{0}(x)+\nabla_{y} u_{1}(x, y):$

$$
\lim _{\varepsilon \rightarrow 0} \int_{\Omega} u_{\varepsilon}(x) \psi\left(x, \frac{x}{\varepsilon}\right) d x=\int_{\Omega} \int_{Y} u_{0}(x) \psi(x, y) d y d x, \quad \forall \psi \in L^{2}\left(\Omega, C_{\sharp}(Y)\right),
$$

$\lim _{\varepsilon \rightarrow 0} \int_{\Omega} \nabla u_{\varepsilon}(x) \Psi\left(x, \frac{x}{\varepsilon}\right) d x=\int_{\Omega} \int_{Y}\left(\nabla_{x} u_{0}(x)+\nabla_{y} u_{1}(x, y)\right) \Psi(x, y) d y d x$,

$$
\forall \Psi \in\left(L^{2}\left(\Omega ; C_{\sharp}(Y)\right)\right)^{N}
$$

\section{Remark 7}

- Two-scale convergence can also extend to periodic surface cases ([8] and [54]). If we note by $\Gamma_{\varepsilon}$ a collection of periodically repeated surfaces in the $\Omega$ domain following the $\varepsilon$ period (the edges of the inclusions present in $\Omega$ for the example in Figure 1), and by $\Gamma$ the corresponding reference surface such as $\forall x \in \Gamma_{\varepsilon} \Rightarrow y=\frac{x}{\varepsilon} \in \Gamma$.

- The Proposition 6, can be expanded to the $L^{p}$ space (see [49], p. 22).

Proposition 7 ([41], p. 34). For all sequence $u_{\varepsilon}$ on $L^{2}\left(\Gamma_{\varepsilon}\right)$ which checks

$$
\varepsilon \int_{\Gamma_{\varepsilon}}\left|u_{\varepsilon}\right|^{2} d x \leqslant C, \quad C>0
$$


there is a subsequence, always noted $u_{\varepsilon}$, and a function $u_{0}(x, y) \in$ $L^{2}\left(\Omega ; L_{\sharp}^{2}(\Gamma)\right)$ such that $u_{\varepsilon}$ two-scale converges to $u_{0}$ in the meaning

$$
\lim _{\varepsilon \rightarrow 0} \int_{\Gamma_{\varepsilon}} u_{\varepsilon}(x) \psi\left(x, \frac{x}{\varepsilon}\right) d x=\int_{\Omega} \int_{\Gamma} u_{0}(x, y) \psi(x, y) d y d x
$$

for any test function Y-periodic $\psi(x, y) \in L^{2}\left(\Omega ; C_{\sharp}(\Gamma)\right)$.

Remark 8 ([41], p. 34). If $u_{\varepsilon}$ is bounded in $L^{2}(\Omega)$ and if $\nabla u_{\varepsilon}$ is bounded in $L^{2}(\Omega)$ as well, using Lemma 4.2.4 in [9], we deduce that $u_{\varepsilon}$ verifies (33) and two-scale converges to a limit $v_{0}$ which is nothing but the trace of $u_{0}$ on $\Gamma, u_{0}$ being the two-scale limit of $u_{\varepsilon}$. $v_{0}$ is noted by $u_{0}$. If $u_{\varepsilon}$ is bounded in $H^{1}(\Omega)$ we have

$$
\begin{gathered}
\lim _{\varepsilon \rightarrow 0} \int_{\Omega} u_{\varepsilon}(x) \psi\left(x, \frac{x}{\varepsilon}\right) d x=\int_{\Omega} \int_{Y} u_{0}(x) \psi(x, y) d y d x, \\
\lim _{\varepsilon \rightarrow 0} \varepsilon \int_{\Gamma_{\varepsilon}} u_{\varepsilon}(x) \psi\left(x, \frac{x}{\varepsilon}\right) d \gamma(x)=\int_{\Omega} \int_{\Gamma} u_{0}(x) \psi(x, y) d \gamma(y) d x, \\
\lim _{\varepsilon \rightarrow 0} \int_{\Omega} \nabla u_{\varepsilon}(x) \cdot \Psi\left(x, \frac{x}{\varepsilon}\right) d x=\int_{\Omega} \int_{Y}\left(\nabla_{x} u_{0}(x)+\nabla_{y} u_{1}(x, y)\right) \\
\cdot \Psi(x, y) d y d x,(35)
\end{gathered}
$$

for all function $\psi(x, y) \in L^{2}\left(\Omega ; C_{\sharp}(Y)\right)$ and any function $\Psi(x, y) \in\left(L^{2}(\Omega\right.$; $\left.\left.C_{\sharp}(Y)\right)\right)^{N}$.

Proposition 8 ([49], p. 22). Let $u_{\varepsilon}$ be a bounded sequence of $W^{1, p}(\Omega)$. Then $u_{\varepsilon}$ converges two-scale, to a function $u_{0}(x) \in W^{1, p}(\Omega)$ and there is a function $u_{1}(x, y) \in L^{p}\left(\Omega ; W_{\sharp}^{1, p}(\Omega)\right)$ such that $\nabla u_{\varepsilon}$ two-scale converges to $\nabla_{x} u_{0}(x)+\nabla_{y} u_{1}(x, y)$. 
Remark 9 ([51], p. 6). For a sequence $\left(u_{\varepsilon}\right)$ in $L^{2}(\Omega)$ space, we have the following relations for the convergences:

Strong convergence $\Rightarrow$ Two-scale convergence $\Rightarrow$ Weak convergence.

Weak convergence $\nRightarrow$ Two-scale convergence $\nRightarrow$ Strong convergence.

The definition of two-scale convergence can take into account time dependence, [50].

Definition 2 ([27], p. 22). Let $\left(u_{\varepsilon}\right)$ be a sequence of functions in $L^{2}((0, T) \times \Omega)$. This sequence two-scale converges to a limit $u \in L^{2}((0, T) \times \Omega \times Y)$ if we have

$$
\begin{gathered}
\forall \psi(t, x, y) \in L^{2}\left((0, T) \times \Omega ; C_{\sharp}(Y)\right), \\
\lim _{\varepsilon \rightarrow 0} \int_{0}^{T} \int_{\Omega} u_{\varepsilon}(t, x) \psi\left(t, x, \frac{x}{\varepsilon}\right) d x d t=\int_{0}^{T} \int_{\Omega} \int_{Y} u(t, x, y) \psi(t, x, y) d y d x d t .
\end{gathered}
$$

The definition quickly expands to a vector or tensor functions valued.

From a similar result in [3], the results are extended to timedependent functions resulting in the following compactness as in [59].

Lemma 3 ([27], p. 22). (a) Each bounded sequence in $L^{2}((0, T) \times \Omega)$ contains a subsequence which two-scale converges to a limit $u \in L^{2}((0, T) \times \Omega \times Y)$.

(b) Let $\left(u_{\varepsilon}\right)$ be a bounded sequence in $L^{2}\left(0, T ; H^{1}(\Omega)\right)$. Then, there exists $u_{0} \in L^{2}\left(0, T ; H^{1}(\Omega)\right)$ and $u_{1} \in L^{2}\left((0, T) \times \Omega ; H_{\sharp}^{1}(Y)\right)$ such that up to a subsequence, $u_{\varepsilon}$ and $\nabla u_{\varepsilon}$ two-scale converges to $u_{0}$ and $\nabla_{x} u_{0}$ $(t, x)+\nabla_{y} u_{1}(t, x, y)$, respectively. 
If in addition $\partial_{t} u_{\varepsilon}$ is bounded in $L^{2}((0, T) \times \Omega)$, then $\partial_{t} u_{0} \in L^{2}$ $((0, T) \times \Omega)$ and $\partial_{t} u_{\varepsilon}$ two-scale converges to $\partial_{t} u_{0}$.

Two-scale convergence is defined in $H^{-1}(0, T)$ as follows:

Definition 3 ([27], p. 37). Let $\left(\phi_{\varepsilon}\right)_{\varepsilon>0}$ be a sequence in $H^{-1}(0, T ; M)$. We say that $\phi_{\varepsilon}$ two-scale converges to $\phi$ in $H^{-1}\left(0, T ; L_{0}^{2}(\Omega \times Y)\right)$ when:

$$
\begin{gathered}
\forall \psi(t, x, y) \in H_{0}^{1}\left((0, T) ; L^{2}\left(\Omega ; C_{\sharp}(Y)\right),\right. \\
\lim _{\varepsilon \rightarrow 0} \int_{0}^{T} \int_{\Omega} \phi_{\varepsilon}(t, x) \psi\left(t, x, \frac{x}{\varepsilon}\right) d x d t=\int_{0}^{T} \int_{\Omega} \int_{Y} \phi(t, x, y) \psi(t, x, y) d y d x d t .
\end{gathered}
$$

Since the time variable is only a parameter in the two-scale convergence defined by (37) and $H^{-1}(0, T)$ is separable, the weak two-scale convergence defined by (37) has the same compactness property as in the square-integrable in time case (Lemma 3).

\subsection{Alternative approaches to the two-scale convergence ([53], pp. 99-100)}

In [13], the authors dealt with a homogenization technique which was used for the description of porous media. It is suitable for an alternative approach to two-scale convergence. The alternative approach is based on the so-called periodic unfolding also called two-scale transform. This idea is based on the so-called transform which changes a sequence of onevariable functions $\left\{u_{\varepsilon}(x)\right\}$ into a sequence of two-variable functions $\left\{\mathcal{T}_{\varepsilon} u(x, y)\right\}$. 
For each $\varepsilon$ let us consider small non-overlapping cubes $C_{\varepsilon}^{k}=\varepsilon Y+\varepsilon k$, $k \in \mathbb{Z}^{N}$. Here, for sake of simplicity, we restrict ourselves to the domains $\Omega$ that can be decomposed into these cubes, i.e., $\bar{\Omega}=\bigcup_{k} \bar{C}_{\varepsilon}^{k}$. The sequence $\left\{\mathcal{I}_{\varepsilon} u(x, y)\right\}$ is defined by the relation

$$
\mathcal{T}_{\varepsilon} u(x, y)=u_{\varepsilon}\left(\varepsilon\left[\frac{x}{\varepsilon}\right]+\varepsilon y\right), \quad x \in \Omega, y \in Y
$$

where $[x]=k$ is the vector of the greatest integers $k_{i}$ less than or equal to $x_{i}$. On each cube $C_{\varepsilon}^{k} \times Y$ the function $\mathcal{T}_{\varepsilon} u$ is constant in the variable $x$ and as a function of $y$ it is the function $u_{\varepsilon}(x)$ on $C_{\varepsilon}^{k}$ transformed onto the unit cube $Y$. The alternative definition reads:

Definition 4. We say that a sequence $\left(u_{\varepsilon}\right)$ in $L^{p}(\Omega)$ two-scale converges to a function $u_{0}(x, y) \in L^{p}(\Omega \times Y)$, if

$$
\lim _{\varepsilon \rightarrow 0} \int_{\Omega} \int_{Y}\left[\mathcal{T}_{\varepsilon} u(x, y)-u_{0}(x, y)\right] \psi(x, y) d y d x=0
$$

for any test function $\quad \varphi \in \mathcal{D}\left(\Omega ; C_{\sharp}^{\infty}(Y)\right)$. Moreover, if $\mathcal{T}_{\varepsilon} u \rightarrow u_{0}$ in $L^{p}(\Omega \times Y)$ strongly, we say that $\left(u_{\varepsilon}\right)$ two-scale converges strongly.

We can refer to the article [29], where the approach mentioned above is also used and is called periodic unfolding. Many authors have applied this approach to the case of periodic multi-scale problems.

The other alternative approach is based on the so-called inverse twoscale transform which changes a sequence $\left\{\bar{\psi}_{\varepsilon}(x)\right\}$ of one-variable functions into a sequence of two-variable functions $\psi(x, y)$. The functions $\bar{\psi}_{\varepsilon}$ are built as follows. Similarly, as in the previous transform, we consider non-overlapping cubes $C_{\varepsilon}^{k}$ that cover the $\Omega$ domain (here the 
$\Omega$ domain does not need a union of $C_{\varepsilon}^{k}$ cubes, i.e., $\left.\bar{\Omega} \subseteq \bigcup_{k} \bar{C}_{\varepsilon}^{k}\right)$. Outside the domain we pose $\psi(x, y)=0$. Let us average the extended function $\psi(x, y)$ with respect to the first variable:

$$
\bar{\psi}_{\varepsilon}(x)=\frac{1}{\varepsilon^{N}} \int_{C_{\varepsilon}^{k}} \psi\left(\xi, \frac{x}{\varepsilon}\right) d \xi, \quad x \in C_{\varepsilon}^{k} .
$$

Definition 5. Let $\left(u_{\varepsilon}\right)$ be a sequence in $L^{p}(\Omega)$. We say that $\left(u_{\varepsilon}\right)$ two-scale converges to a function $u_{0} \in L^{p}(\Omega \times Y)$, if

$$
\lim _{\varepsilon \rightarrow 0} \int_{\Omega} u_{\varepsilon}(x) \bar{\psi}_{\varepsilon}(x) d x=\int_{\Omega} \int_{Y} u_{0}(x, y) \psi(x, y) d y d x
$$

for each test function $\psi(x, y) \in \mathcal{D}\left(\Omega ; C_{\sharp}^{\infty}(Y)\right)$. Moreover, if

$$
\lim _{\varepsilon \rightarrow 0}\left\|u_{\varepsilon}(x)-\bar{u}_{0}^{\varepsilon}(x)\right\|_{L^{p}(\Omega)}=0, \text { where } \bar{u}_{0}^{\varepsilon}(x)=\frac{1}{\varepsilon^{N}} \int_{C_{\varepsilon}^{k}} u_{0}\left(\xi, \frac{x}{\varepsilon}\right) d \xi, \quad x \in C_{\varepsilon}^{k}
$$

we say that $\left(u_{\varepsilon}\right)$ two-scale converges strongly.

Remark 10. The two-scale transformation used in the Definition 4 allows us to define two-scale convergence and strong two-scale convergence more naturally with the help of weak convergence with regular test functions. On the other hand, the Definition 5 is similar to the classic definition by Nguetseng and Allaire, but differs from (30) in the choice of test function on the left side.

Why do we look for alternative approaches to the original one? In the definition we want to test the convergence with functions from a space as small as possible, thus, smooth functions are convenient. On the other hand, in applications the largest class is desirable. In Definition 1 we cannot take the test functions $\psi(x, y)$ from the whole space $L^{q}(\Omega \times Y)$, since it is not correctly defined on the zero-measure set $\{[x, y]: y=x / \varepsilon\}$ and thus the measurability of the composed function $\psi(x, x / \varepsilon)$ is not 
guaranteed. Moreover, the test functions can satisfy some convergences, which is not always obvious. The class of suitable test functions is discussed at the top of the following section. Such functions are called admissible. Further, we will see that the two mentioned alternative definitions avoid described problems.

The main result of convergence on two-scale is convergence into a norm.

Theorem 3 ([49], p. 10). Let $\left(u_{\varepsilon}\right)_{\varepsilon>0}$ be a sequence in $L^{p}(\Omega)$ which two-scale converges to $u_{0} \in L^{p}(\Omega \times Y)$. Then

$$
u_{\varepsilon} \rightarrow v(x)=\int_{Y} u_{0}(x, y) d y \quad \text { weakly in } L^{p}(\Omega),
$$

and $\left(u_{\varepsilon}\right)$ is bounded.

Theorem 4 ([49], p. 17). Let $\left(u_{\varepsilon}\right)_{\varepsilon>0}$ be a sequence in $L^{p}(\Omega)$ which two-scale converges to $u_{0} \in L^{p}(\Omega \times Y)$. Then

$$
\lim _{\varepsilon \rightarrow 0} \inf \left\|u_{\varepsilon}\right\|_{L^{p}(\Omega)} \geqslant\left\|u_{0}\right\|_{L^{p}(\Omega \times Y)} \geqslant\|u\|_{L^{p}(\Omega)}
$$

where $u(x)=\int_{Y} u_{0}(x, y) d y$.

Remark 11. We note that the result (42) of the Theorem 4 can be compared with the well-known fact that if $u_{\varepsilon} \rightarrow u$ weakly, then

$$
\lim _{\varepsilon \rightarrow 0} \inf \left\|u_{\varepsilon}\right\|_{L^{p}(\Omega)} \geqslant\|u\|_{L^{p}(\Omega)}
$$

So far, we have given the main results on the bounded sequences of $L^{p}(\Omega)$. For bounded sequences of $H^{1}(\Omega)$, the results may be more accurate. 
Theorem 5 ([10], pp. 23). Let $\left(u_{\varepsilon}\right)_{\varepsilon>0}$ be a bounded sequence in $H^{1}(\Omega)$. Then, there exists $u_{0} \in H^{1}(\Omega)$ and $u_{1} \in L^{2}\left(\Omega, H_{\sharp}^{1}(Y)\right)$, such that, up to the extraction of a subsequence, one has

$u_{\varepsilon}$ converges to $u_{0}$ weakly in $H^{1}(\Omega)$,

$u_{\varepsilon}$ converges to $u_{0}$ strongly in $L^{2}(\Omega)$,

$u_{\varepsilon}$ two-scale converges to $u_{0}$,

$\nabla u_{\varepsilon}$ two-scale converges to $\nabla_{x} u_{0}(x)+\nabla_{y} u_{1}(x, y)$.

Proposition 9 ([10], pp. 26). Let $\left(u_{\varepsilon}\right)_{\varepsilon>0}$ be a bounded sequence of $L^{2}(\Omega)$ such that $\left(\varepsilon \nabla u_{\varepsilon}\right)_{\varepsilon>0}$ is bounded in $L^{2}(\Omega)$. There exists $u_{0}(x, y) \in L^{2}(\Omega \times Y)$, such that, up to the extraction of a subsequence, one has

$u_{\varepsilon}$ two-scale converges to $u_{0}$,

$\varepsilon \nabla u_{\varepsilon}$ two-scale converges to $\nabla_{y} u_{0}(x, y)$.

Theorem 6 ([49], p. 20). Any function $u \in L^{p}(\Omega \times Y)$ is attained as a two-scale limit.

\subsection{Other properties of two-scale convergence ([53], p. 107-108)}

The sequences from the previous two properties (4) and (5) point to an interesting fact. An extracted subsequence of weakly converging sequence converges to the same limit. In the case of two-scale convergence, we must consider convergence also with respect to subsequences of periods. Otherwise the limits may differ (e.g., the sequence $\left(v_{\varepsilon}\right)$ from the properties above can be considered as extracted subsequence from $\left.\left(u_{\varepsilon}\right)\right)$. 
Definition 6. Let $u \in L^{p}(\Omega \times Y)$, we define $\mathcal{T}_{\varepsilon}^{*}$ by

$$
\left(\mathcal{T}_{\varepsilon}^{*} u\right)(x, y)= \begin{cases}u\left(\varepsilon\left[\frac{x}{\varepsilon}\right]_{Y}+\varepsilon y\right), & \text { for } x \in \Omega_{\varepsilon}, \\ u(x), & \text { for } x \in \Lambda_{\varepsilon}, \text { where } \Lambda_{\varepsilon}=\Omega-\Omega_{\varepsilon} .\end{cases}
$$

In the case where $\Omega=\mathbb{R}^{N}$ or if the domain $\Omega$ can be written as interior of a union of complete cells we can take $\Omega_{\varepsilon}=\Omega$ and $\Lambda_{\varepsilon}=\emptyset$.

Property 1 ([39], p. 78). Let $f, g \in L^{p}(\Omega)$ and $\psi \in L^{\infty}\left(Y_{\sharp}\right)$, such that $\int_{Y} \psi(y) d y=0$.

Then the sequence $u_{\varepsilon}(x)=f(x) \psi\left(\frac{x}{\varepsilon}\right)+g(x)$ is bounded in $L^{p}(\Omega)$. Since its unfolding $\mathcal{T}_{\varepsilon}^{*} u_{\varepsilon}$ yields $\left(\mathcal{T}_{\varepsilon}^{*} u_{\varepsilon}\right)(x, y)=f(x) \psi(y)+g(x)$ in $\Omega_{\varepsilon} \times Y$. It strongly two-scale converges in $L^{p}(\Omega)$ to the limit $u_{0}(x, y)=$ $f(x) \psi(y)+g(x)$. The sequence $u_{\varepsilon}$ converges to $g(x)$ in $L^{p}(\Omega)$ weakly, but not strongly, unless that $f(x) \equiv 0$ or $\psi(y) \equiv 0$. The property shows that the local oscillations of $u_{\varepsilon}$, which are lost in the usual weak limit $L^{p}(\Omega)$, are conserved in the strong two-scale limit.

Remark 12. In the previous property (4), the sequence was strongly two-scale converging. It was caused by the fact that the period $\varepsilon$ of $\phi\left(\frac{x}{\varepsilon}\right)$ was "in resonance" with the scale $E=\{\varepsilon\}$. Modifying the sequence to $u_{\varepsilon}(x)=f(x) \psi\left(\frac{2 x}{\varepsilon}\right)+g(x)$ with the same scale $E$, the sequence also strongly two-scale converge but the limit is $u_{0}(x, y)=f(x) \varphi(2 y)+g(x)$. The weak $L^{p}(\Omega)$ limit is unchanged. 
If the period of the function $\psi$ is not "in resonance" with the $E$ scale,

i.e., their ratio is irrational, e.g., $u_{\varepsilon}(x)=f(x) \psi\left(\frac{x}{\sqrt{2} \varepsilon}\right)+g(x)$, then the sequence $u_{\varepsilon}$ does not converge two-scale strongly but only weakly. Its limit $u_{0}(x, y)=g(x)$ is independent of $y$, i.e., the local oscillations are again lost.

\section{Solving Some Problems by Two-Scale Convergence Method}

We will give in this section some problems solved with two-scale convergence and applied it to an example.

\subsection{Some problems solved by the two-scale convergence method}

Two-scale convergence has been used to study different problems. We give an overview of these problems.

\section{Two-scale convergence applied to the Vlasov-Poisson equation and Vlasov equation}

Jiann-Sheng Jiang and Chi-Kun Lin use two-scale convergence in their article "Weak turbulence plasma induced by two-scale homogenization", see [46]. They showed that the homogenization limit, in the sense of two-scale limit, of the distribution function satisfies the linear Vlasov-Poisson equations. Moreover, the limit distribution function can be decomposed into the mean and the fluctuation parts. They also investigate the Landau damping from the point of view of homogenization through the two-scale limit.

They use the two-scale convergence on the quasi-linear theory of the plasma turbulence described by the Vlasov-Poisson system.

In the same topic Aurore Back and Emmanuel Frenod use it in their article "Geometric two-scale convergence on manifold and applications to the Vlasov equation", see [17]. They adapt the two-scale convergence on manifold using the Birkhoff's theorem and they do asymptotic analysis using geometric objects (differential forms). 


\section{Two-scale convergence applied to fluid flow in porous media}

Gregoire Allaire applied the two-scale convergence to fluid flow in porous media, see ([4], pp. 13-19). He considered the steady Stokes equations in a porous medium $\Omega_{\varepsilon}$ with a Dirichlet boundary condition. Denoting by $u_{\varepsilon}$ and $p_{\varepsilon}$ the velocity and pressure of the fluid, and $f$ the density of forces acting on the fluid. The system of equations is

$$
\begin{cases}\nabla p_{\varepsilon}-\varepsilon^{2} \Delta u_{\varepsilon}=f & \text { in } \Omega_{\varepsilon}, \\ \operatorname{div} u_{\varepsilon}=0 & \text { in } \Omega_{\varepsilon}, \\ u_{\varepsilon}=0 & \text { on } \partial \Omega_{\varepsilon} .\end{cases}
$$

Two-scale convergence applied to behaviour of the warping and the torsion problems

Polisevski and Mascarenhas in [61] use the two-scale convergence method to study the homogenized behaviour of the warping and the torsion problems in a two-dimensional domain with a quasi-periodic perforation, as well as the relationship between the two homogenized problems. The Neumann problem was also analyzed.

\section{Two-scale convergence applied to the stochastic}

Wright et al. in [23] studied stochastic homogenization of partial differential equations and generalise the method of two-scale convergence. The two-scale convergence has been extended from the periodic setting to the more general stochastic setting. The compactness and convergence results are important tools in the resolution of homogenization problems for partial differential equation. These results can be applied to the monotone operators and nonlocal. For the two-scale convergence of almost periodic function, see [26]. 


\section{Two-scale convergence applied to the viscous fluid}

Saint Jean-Paulin and Polisevski in [60] used the two-scale convergence in the study of viscous fluid. The so-called dual-porosity model for immiscible incompressible two-phase flow in a natural fractured reservoir has been studied in the means of two-scale convergence method by Bourgeat et al. in [22].

\section{Two-scale convergence on BV-spaces}

The two-scale convergence method has been used by Amar in [11] to study some classes of homogenization problems on BV-spaces for periodic energies with linear growth.

\section{Two-scale convergence applied to other problems}

The two-scale limit has many others applications. It can also be applied to fluid flow problems, see [2, 12, 20, 21, 34, 38, 62], and to several other types of homogenized problems. For magnetic field problems, see [40, 64] and for parabolic problems, see [25, 44, 65]. For degenerated parabolic differential equations, see [32, 33, 52]. For spectral problems in fluid-solid structures, see [7]. Other applications of two-scale convergence can be found in $[1,48,50]$ and especially the books $[31,35]$.

Other methods of analyzing multi-scale problems exist. One of these methods is the Representative Elementary Volume method (VER or RVE) [28, 42, 43].

The basic idea is to calculate means of disturbances in a volume representative of the heterogeneity of the system, then use these means to describe macroscopically the problem initially described in a microscopic way.

The method of mathematical homogenization is based on the same principle. Nevertheless, the averages it provides are obtained in a mathematically more rigorous and justified manner. Moreover, it allows to approach the local values of the disturbances thanks to corrective

terms. The physical homogenization method is limited to the definition of an average value of these disturbances. 


\subsection{Applications to 2nd order elliptic linear equations ([10], pp. 27)}

We now turn to the homogenization of the model problem and generalize the method to classical second order elliptic PDEs. As we shall see, the main strategy consists in the following methodology:

- find a sequence $\left(u_{\varepsilon}\right)_{\varepsilon}$ bounded in $H^{1}$;

- extract a subsequence and apply the Theorem 5;

- identify the problems solved by $u_{0}$ and $u_{1}$;

- show that the whole sequence converges;

- give sufficient conditions to get the strong convergence and prove the multiscale expansion. Let's consider the problem (2), namely,

$$
\begin{cases}-\operatorname{div}\left(A\left(\frac{x}{\varepsilon}\right) \nabla u_{\varepsilon}(x)\right) & =f(x) \text { in } \Omega \\ u_{\varepsilon} & =0 \text { on } \partial \Omega\end{cases}
$$

where $\Omega$ is a bounded open set. Note that under the classic tensor assumptions of $A$, namely, the existence of $0<c<C$ such that

$$
\forall \xi \in \mathbb{R}^{N}, c|\xi|^{2} \leqslant(A \xi, \xi) \leqslant C|\xi|^{2},
$$

and the fact that $f \in L^{2}(\Omega)$, the problem (2) admits a unique solution $u_{\varepsilon} \in H_{0}^{1}(\Omega)$, which moreover satisfies

$$
\begin{aligned}
-\int_{\Omega} \operatorname{div}\left(A\left(\frac{x}{\varepsilon}\right) \nabla u_{\varepsilon}(x)\right) \cdot u_{\varepsilon}(x)= & -\int_{\partial \Omega}\left(A\left(\frac{x}{\varepsilon}\right) \nabla u_{\varepsilon}(x)\right) \cdot u_{\varepsilon}(x) \cdot n(x) d \sigma \\
& +\int_{\Omega}\left(A\left(\frac{x}{\varepsilon}\right) \nabla u_{\varepsilon}(x)\right) \cdot \nabla u_{\varepsilon}(x) d x \\
= & \int_{\Omega}\left(A\left(\frac{x}{\varepsilon}\right) \nabla u_{\varepsilon}(x)\right) \cdot \nabla u_{\varepsilon}(x) d x \\
= & \int_{\Omega}\left(A\left(\frac{x}{\varepsilon}\right) \nabla u_{\varepsilon}(x), \nabla u_{\varepsilon}(x)\right) d x .
\end{aligned}
$$


The following variational formulation:

$$
\begin{cases}\int_{\Omega}\left(A\left(\frac{x}{\varepsilon}\right) \nabla u_{\varepsilon}(x), \nabla u_{\varepsilon}(x)\right) d x & =\int_{\Omega} f(x) u_{\varepsilon}(x) d x \text { in } \Omega \\ u_{\varepsilon} & =0 \text { on } \partial \Omega\end{cases}
$$

lead to

$$
\begin{aligned}
\int_{\Omega}\left(A\left(\frac{x}{\varepsilon}\right) \nabla u_{\varepsilon}(x), \nabla u_{\varepsilon}(x)\right) d x & =\int_{\Omega} f(x) u_{\varepsilon}(x) d x \\
& \leqslant\|f\|_{L^{2}}\left\|u_{\varepsilon}\right\|_{L^{2}} \\
& \leqslant C_{P}\|f\|_{L^{2}}\left\|\nabla u_{\varepsilon}\right\|_{L^{2}},
\end{aligned}
$$

where $C_{P}$ represents Poincaré's constant on $\Omega$. Using the coercivity hypothesis (44), we easily deduce

$$
\left(A\left(\frac{x}{\varepsilon}\right) \nabla u_{\varepsilon}(x), \nabla u_{\varepsilon}(x)\right)_{0, \Omega}=\int_{\Omega}\left(A\left(\frac{x}{\varepsilon}\right) \nabla u_{\varepsilon}(x), \nabla u_{\varepsilon}(x)\right) d x,
$$

or

$$
c\left\|\nabla u_{\varepsilon}\right\|_{L^{2}}^{2} \leqslant\left(A\left(\frac{x}{\varepsilon}\right) \nabla u_{\varepsilon}(x), \nabla u_{\varepsilon}(x)\right)_{0, \Omega} \leqslant C_{P}\|f\|_{L^{2}}\left\|\nabla u_{\varepsilon}\right\|_{L^{2}}
$$

Then

$$
\begin{aligned}
\left\|\nabla u_{\varepsilon}\right\|_{L^{2}} & \leqslant \frac{C_{P}}{c}\|f\|_{L^{2}} \\
& \lesssim\|f\|_{L^{2}} .
\end{aligned}
$$

We have the following theorem.

Theorem 7 ([49], p. 25). The solution sequence of the Equation (45) weakly converges to $u_{0}$ in $W_{0}^{1,2}(\Omega)$ and the sequence $\nabla u_{\varepsilon}$ two-scale converges to $\nabla u_{0}(x)+\nabla_{y} u_{1}(x, y)$, where $\left(u_{0}, u_{1}\right)$ is the single solution in $W_{0}^{1,2}(\Omega) \times L^{2}\left(\Omega, W_{\sharp}^{1,2}(Y)\right)$ of the homogenized equation: 


$$
\begin{aligned}
& \int_{\Omega} \int_{Y}\left(A(y)\left(\nabla u_{0}(x)+\nabla_{y} u_{1}(x, y)\right), \nabla v(x)+\nabla_{y} v_{1}(x, y)\right) d y d x=\int_{\Omega} f v d x, \\
& \text { for all } v(x) \in W_{0}^{1,2}(\Omega) \text { and } v_{1}(x, y) \in L^{2}\left(\Omega, W_{\sharp}^{1,2}(Y)\right) .
\end{aligned}
$$

\section{Conclusion}

The theory of homogenization uses the method of asymptotic analysis to describe the behaviour of a physics system. A new method of convergence of homogenization processes is proposed to justify the theory of homogenization: two-scale convergence. In this article we surveyed some phenomena in the two-scale convergence. By the original definitions from Nguetseng we give again some other definitions. The two-scale convergence has been genaralized to the multiscale convergence, see [6] and Corollary 1.16 of [3]. We also see that the twoscale convergence can be defined by a new way: the alternative approaches. This principle is to transform a sequence of one variable function to a sequence of two variable functions. We give some examples to help the reader to more understand the notion of two-scale convergence. We see also that the choice of the space is very important to be able to apply the definition of two-scale convergence.

\section{Acknowledgement}

We thank Professor Gabriel Nguetseng for the help that he gave us to more understand the notion of two-scale convergence. The help he gave us with the Lemma 2, the Proposition 2 and Proposition 3 have been very fructful and this has helped us to give the Examples 1 and 2. We thank Professor Nakoulima for the different discussions we had together. The time spent working with him allowed us to better understand the subject. And his contribution was very important for Examples 1 and 2. Our thanks again to Professor Gregoire Allaire for some directions he give us. 


\section{References}

[1] R. Alexandre, Homogenisation and $\theta-2$ convergence, Proceedings of the Royal Society of Edinburgh Section A: Mathematics 127(3) (1997), 441-455.

DOI: https://doi.org/10.1017/S0308210500029863

[2] G. Allaire, Homogenization of the Unsteady Stokes Equations in Porous Media, Progress in Partial Differential Equations: Calculus of Variations, Applications, Pont-à-Mousson, 1991.

[3] G. Allaire, Homogenization and two-scale convergence, SIAM Journal on Mathematical Analysis 23(6) (1992), 1482-1518.

DOI: https://doi.org/10.1137/0523084

[4] G. Allaire, Two-Scale Convergence and Homogenization of Periodic Structures, 1993.

[5] G. Allaire, Introduction to Homogenization Theory, VKI Lecture Series 2001-2002 Multiscale Methods, Lecture 1, 2002.

[6] G. Allaire and M. Briane, Multiscale convergence and reiterated homogenisation, Proceedings of the Royal Society of Edinburgh Section A: Mathematics 126(2) (1996), 297-342.

DOI: https://doi.org/10.1017/S0308210500022757

[7] G. Allaire and C. Conca, Boundary layers in the homogenization of a spectral problem in fluid-solid structures, SIAM Journal on Mathematical Analysis 29(2) (1998), 343-379.

DOI: https://doi.org/10.1137/S0036141096304328

[8] G. Allaire, A. Damlamian and U. Hornung, Two-scale convergence on periodic surfaces and applications, In Proceedings of the International Conference on Mathematical Modelling of Flow Through Porous Media (May 1995), A. Bourgeat et al., Editors, (1996), 15-25.

[9] G. Allaire and K. El Ganaoui, Homogenization of a conductive and radiative heat transfer problem, Multiscale Modeling \& Simulation 7(3) (2008), 1148-1170.

DOI: https://doi.org/10.1137/080714737

[10] F. Alouges, Introduction to Periodic Homogenization, Minicourse in Tohoku University 4 (2016).

http://www.cmap.polytechnique.fr/ alouges/Homogenization/CoursHomog.pdf

[11] M. Amar, Two-scale convergence and homogenization on BV( $\Omega)$, Asymptotic Analysis 16(1) (1998), 65-84.

[12] S. N. Antontsev, A. M. Meirmanov and V. V. Yurinsky, Homogenization of stokestype equations with variable viscosity, Siberian Advances in Mathematics 8(2) (1998), 1-29. 
[13] T. Arbogast, J. Douglas and U. Hornung, Derivation of the double porosity model of single phase flow via homogenization theory, SIAM Journal on Mathematical Analysis 21(4) (1990), 823-836.

DOI: https://doi.org/10.1137/0521046

[14] I. Babuska, Solution of problems with interfaces and singularities, In Symposium on Mathematical Aspects of Finite Elements in Partial Differential Equations Academic Press, New York (1974), pages 213-277.

DOI: https://doi.org/10.1016/B978-0-12-208350-1.50013-3

[15] I. Babuska, Homogenization approach in engineering, Computing Methods in Applied Sciences and Engineering (1975), 137-153.

DOI: https://doi.org/10.1007/978-3-642-85972-4_8

[16a] I. Babuska, Solution of interface problems by homogenization: I, SIAM Journal on Mathematical Analysis 7(5) (1976), 603-634.

DOI: https://doi.org/10.1137/0507048

[16b] I. Babuska, Solution of interface problems by homogenization: II, SIAM Journal on Mathematical Analysis 7(5) (1976), 635-645.

DOI: https://doi.org/10.1137/0507049

[16c] I. Babuska, Solution of interface problems by homogenization: III, SIAM Journal on Mathematical Analysis 8(6) (1977), 923-937.

DOI: https://doi.org/10.1137/0508071

[17] A. Back and E. Frenod, Geometric two-scale convergence on manifold and applications to the vlasov equation, Discrete and Continuous Dynamical Systems Series S 8(1) (2015), 223-241.

DOI: https://doi.org/10.3934/dcdss.2015.8.223

[18] N. Bakhvalov and G. Panasenko, Homogenisation: Averaging Processes in Periodic Media, Kluwer, Dordrecht, 1989.

[19] A. Bensoussan, J. L. Lions and G. Papanicolaou, Asymptotic Analysis for Periodic Structures, Volume 5 of Studies in Mathematics and its Applications, North-Holland Publishing Co., Amsterdam, 1978.

[20] W. Bielski, J. J. Telega and R. Wojnar, Nonstationary flow of a viscous fluid through a porous elastic medium: Asymptotic analysis and two-scale convergence, Mechanics Research Communications 26(5) (1999), 619-628.

DOI: https://doi.org/10.1016/S0093-6413(99)00070-1

[21] A. Bourgeat and A. Hidanl, Effective model of two-phase flow in a porous medium made of different rock types, Applicable Analysis 58(1-2) (1995), 1-29.

DOI: https://doi.org/10.1080/00036819508840360 
[22] A. Bourgeat, S. Luckhaus and A. Mikelic, Convergence of the homogenization process for a double-porosity model of immiscible two-phase flow, SIAM Journal on Mathematical Analysis 27(6) (1996), 1520-1543.

DOI: https://doi.org/10.1137/S0036141094276457

[23] S. Wright, A. Mikelic and A. Bourgeat, Stochastic two-scale convergence in the mean and applications, Journal für die Reine und Angewandte Mathematik 456 (1994), 19-51.

DOI: https://doi.org/10.1515/crll.1994.456.19

[24] D. Caillerie, Homogénéisation des matériaux à structure périodique, Méthodes Asymptotiques en Mécanique.

http://mam.ida.upmc.fr/Telechargements/cours-Caillerie.pdf

[25] É. Canon and J. N. Pernin, Homogenization of Diffusion in a Composite Medium with Interfacial Barrier, Comptes Rendus de l'Académie des Sciences, Series I: Mathematics 325(1) (1997), 123-126.

DOI: https://doi.org/10.1016/S0764-4442(97)83946-8

[26] J. Casado-Diaz and I. Gayte, A general compactness result and its application to the two-scale convergence of almost periodic functions, Comptes Rendus de l'Académie des Sciences, Series I: Mathematics 323(4) (1996), 329-334.

[27] P. Cazeaux and C. Grandmont, Homogenization of a multiscale viscoelastic model with nonlocal damping, application to the human lungs, Mathematical Models and Methods in Applied Sciences 25(6) (2015), 1125-1177.

DOI: https://doi.org/10.1142/S0218202515500293

[28] R. M. Christensen, Mechanics of Composite Materials, John Wiley, New-York, 1979.

[29] D. Cioranescu, A. Damlamian and G. Griso, Periodic unfolding and homogenization, Comptes Rendus Mathematique 335(1) (2002), 99-104.

DOI: https://doi.org/10.1016/S1631-073X(02)02429-9

[30] D. Cioranescu, A. Damlamian and G. Griso, The periodic unfolding method in homogenization, SIAM Journal on Mathematical Analysis 40(4) (2008), 1585-1620.

DOI: https://doi.org/10.1137/080713148

[31] D. Cioranescu and P. Donato, An Introduction to Homogenization, Oxford, 1999.

[32] G. W. Clark and L. A. Packer, Two-scale homogenization of implicit degenerate evolution equations, Journal of Mathematical Analysis and Applications 214(2) (1997), 420-438.

DOI: https://doi.org/10.1006/jmaa.1997.5577 
[33] G. W. Clark and L. A. Packer, Two-scale homogenization of non-linear degenerate evolution equations, Journal of Mathematical Analysis and Applications 238(1) (1999), 316-328.

DOI: https://doi.org/10.1006/jmaa.1999.6547

[34] G. W. Clark and R. E. Showalter, Two-scale convergence of a model for flow in a partially fissured medium, Electronic Journal of Differential Equations (1999), 1-20; Article 2.

[35] C. Conca, J. Planchard and M. Vanninathan, Fluids and Periodic Structures, RAM: Research in Applied Mathematics, 1995.

[36] D. Cioranescu, P. Donato and R. Zaki, Periodic unfolding and robin problems in perforated domains, Comptes Rendus Mathematique 342(7) (2006), 469-474.

DOI: https://doi.org/10.1016/j.crma.2006.01.028

[37] A. Ene and J. Saint Jean Paulin, On a model of fractured porous media, Mathematical Modelling of Flow Through Porous Media (1995), pages 402-409.

[38] I. A. Ene and J. Saint Paulin, Homogenization and two-scale convergence for a Stokes or Navier-Stokes flow in an elastic thin porous medium, Mathematical Models and Methods in Applied Sciences 6(7) (1996), 941-955.

DOI: https://doi.org/10.1142/S0218202596000389

[39] Jan Francu, On two-scale convergence and periodic unfolding, Tatra Mountains Mathematical Publications 48(1) (2011), 73-81.

DOI: https://doi.org/10.2478/v10127-011-0007-3

[40] E. Frénod and E. Sonnendrucker, Long time behavior of the two-dimensional Vlasov equation with a strong external magnetic field, Mathematical Models and Methods in Applied Sciences 10(4) (2000), 539-553.

DOI: https://doi.org/10.1142/S021820250000029X

[41] Z. Habibi, Homogenization and Two Scales Convergence of Some Stationary and Non-stationary Heat Transfer Problems, Application to Gas Cooled Nuclear Rectors, PhD Thesis, École Polytechnique, Paris, 2011.

[42] Z. Hashin, The elastic moduli of heterogeneous materials, Journal of Applied Mechanics 29(1) (1962), 143-150.

DOI: https://doi.org/10.1115/1.3636446

[43] Z. Hashin, Analysis of composite materials: A survey, Journal of Applied Mechanics 50(3) (1983), 481-505.

DOI: https://doi.org/10.1115/1.3167081

[44] A. Holmbom, Homogenization of parabolic equations an alternative approach and some corrector-type results, Applications of Mathematics 42(5) (1997), 321-343.

DOI: https://doi.org/10.1023/A:1023049608047 
[45] U. Hornung, Homogenization and Porous Media, Springer Verlag, 1997.

[46] J. Jiang and C. Lin, Weak turbulence plasma induced by two-scale homogenization, Journal of Mathematical Analysis and Applications 410(2) (2014), 585-596.

DOI: https://doi.org/10.1016/j.jmaa.2013.08.010

[47] S. Kesavan, Homogenization of elliptic eigenvalue problems: Part 2, Applied Mathematics and Optimization 5(1) (1979), 197-216.

DOI: https://doi.org/10.1007/BF01442554

[48] R. Lipton and B. Vernescu, Homogenisation of two-phase emulsions, Proceedings of the Royal Society of Edinburgh Section A: Mathematics 124(6) (1994), 1119-1134.

DOI: https://doi.org/10.1017/S0308210500030146

[49] D. Lukkassen, G. Nguetseng and P. Wall, Two-scale convergence, International Journal of Pure and Applied Mathematics 2(1) (2002), 35-86.

[50] R. E. Miller, Homogenization of time-dependent systems with Kelvin-Voigt damping by two-scale convergence, Discrete \& Continuous Dynamical Systems: A 1(4) (1995), 485-502.

DOI: https://doi.org/10.3934/dcds.1995.1.485

[51] A. K. Nandakumaran and S. Sivaji Ganesh, Lectures on two scale convergence and homogenization, In NPDE-TCA Advanced Workshop on Homogenization, Control Theory and Applications, 2016.

[52] A. K. Nandakumaran and M. Rajesh, Homogenization of a nonlinear degenerate parabolic differential equation, Electronic Journal of Differential Equations (2001), 1-19; Article 17.

[53] L. Nechvátal, Alternative approaches to the two-scale convergence, Applications of Mathematics 49(2) (2004), 97-110.

DOI: https://doi.org/10.1023/B:APOM.0000027218.04167.9b

[54] M. Neuss-Radu, Some extensions of two-scale convergence, Comptes Rendus de l'Académie des Sciences, Series I: Mathematics 322(9) (1996), 899-904.

[55] G. Nguetseng, A general convergence result for a functional related to the theory of homogenization, SIAM Journal on Mathematical Analysis 20(3) (1989), 608-623.

DOI: https://doi.org/10.1137/0520043

[56] G. Nguetseng, Homogenization structures and applications I, Zeitschrift für Analysis und Ihre Anwendungen 22(1) (2003), 73-107.

DOI: https://doi.org/10.4171/zaa/1133

[57] G. Nguetseng, Homogenization structures and applications II, Zeitschrift für Analysis und Ihre Anwendungen 23(3) (2004), 483-508.

DOI: https://doi.org/10.4171/zaa/1208 
[58] G. Nguetseng and N. Svanstedt, $\sum$-convergence, Banach Journal of Mathematical Analysis 5(1) (2011), 101-135.

[59] R. P. Gilbert and A. Mikelic, Homogenizing the acoustic properties of the seabed: Part 1, Nonlinear Analysis: Theory, Methods \& Applications 40(1-8) (2000), 185-212.

DOI: https://doi.org/10.1016/S0362-546X(00)85011-7

[60] D. Polisevski and J. Saint Jean-Paulin, Homogenization of stokes-Boussinesq flows in a quasiperiodic domain, Revue Roumaine de Mathématique Pures et Appliquées 40(9-10) (1995), 797-808.

[61] M. L. Mascarenhas and D. Polisevski, The warping, the torsion and the Neumann problems in a quasi-periodically perforated domain, Mathematical Modelling and Numerical Analysis 28(1) (1994), 37-57.

DOI: https://doi.org/10.1051/m2an/1994280100371

[62] M. Rajesh, Correctors for flow in a partially fissured medium, Electronic Journal of Differential Equations (1999), 1-15; Article 27.

[63] E. Sanchez-Palencia, Non-Homogeneous Media and Vibration Theory, Springer, 1980.

[64] N. Wellander, Homogenization of the Maxwell equations: Case I, linear theory, Applications of Mathematics 46(1) (2001), 29-51.

DOI: https://doi.org/10.1023/A:1013727504393

[65] X. Zhang, Two-scale convergence and homogenization for a class of quasilinear elliptic equations, Journal of Partial Differential Equations 9(3) (1996), 263-276. 